\title{
A replication-competent adenovirus-vectored influenza vaccine induces durable systemic and mucosal immunity
}

\author{
Kenta Matsuda, ${ }^{1}$ Stephen A. Migueles, ${ }^{1}$ Jinghe Huang, ${ }^{1}$ Lyuba Bolkhovitinov, ${ }^{1}$ Sarah Stuccio, ${ }^{1}$ Trevor Griesman, ${ }^{1}$ Alyssa A. Pullano, \\ Byong H Kang, ${ }^{1}$ Elise Ishida, ${ }^{1}$ Matthew Zimmerman, ${ }^{1}$ Neena Kashyap, ${ }^{1}$ Kelly M. Martins, ${ }^{1}$ Daniel Stadlbauer, ${ }^{2}$ Jessica Pederson, ${ }^{1}$ \\ Andy Patamawenu, ${ }^{1}$ Nathaniel Wright, ${ }^{1}$ Tulley Shofner, ${ }^{1}$ Sean Evans, ${ }^{1}$ C. Jason Liang, ${ }^{3}$ Julián Candia, ${ }^{4}$ Angelique Biancotto, ${ }^{4}$ \\ Giovanna Fantoni, ${ }^{4}$ April Poole, ${ }^{1}$ Jon Smith, ${ }^{5}$ Jeff Alexander, ${ }^{5}$ Marc Gurwith, ${ }^{5}$ Florian Krammer, ${ }^{2}$ and Mark Connors ${ }^{1}$ \\ ${ }^{1} \mathrm{HIV}$-Specific Immunity Section of the Laboratory of Immunoregulation, National Institute of Allergy and Infectious Diseases (NIAID), NIH, Bethesda, Maryland, USA. ${ }^{2}$ Department of Microbiology, Icahn \\ School of Medicine at Mount Sinai, New York, New York, USA. ${ }^{3}$ Biostatistics Research Branch, NIAID and ${ }^{4}$ Trans-NIH Center for Human Immunology, Autoimmunity, and Inflammation, NIH, Bethesda, \\ Maryland, USA. ${ }^{5}$ Emergent Biosolutions Inc., Gaithersburg, Maryland, USA.
}

\begin{abstract}
BACKCROUND. To understand the features of a replicating vaccine that might drive potent and durable immune responses to transgene-encoded antigens, we tested a replication-competent adenovirus type 4 encoding influenza virus H5 HA (Ad4-H5Vtn) administered as an oral capsule or via tonsillar swab or nasal spray.
\end{abstract}

METHODS. Viral shedding from the nose, mouth, and rectum was measured by PCR and culturing. H5-specific IgC and IgA antibodies were measured by bead array binding assays. Serum antibodies were measured by a pseudovirus entry inhibition, microneutralization, and HA inhibition assays.

RESULTS. Ad4-H5-Vtn DNA was shed from most upper respiratory tract-immunized (URT-immunized) volunteers for 2 to 4 weeks, but cultured from only $60 \%$ of participants, with a median duration of 1 day. Ad4-H5-Vtn vaccination induced increases in $\mathrm{H5}$-specific $\mathrm{CD4}^{+}$and $\mathrm{CD8}^{+} \mathrm{T}$ cells in the peripheral blood as well as increases in IgC and IgA in nasal, cervical, and rectal secretions. URT immunizations induced high levels of serum neutralizing antibodies (NAbs) against H5 that remained stable out to week 26. The duration of viral shedding correlated with the magnitude of the NAb response at week 26. Adverse events (AEs) were mild, and peak NAb titers were associated with overall AE frequency and duration. Serum NAb titers could be boosted to very high levels 2 to 5 years after Ad4-H5-Vtn vaccination with recombinant $\mathrm{H} 5$ or inactivated split H5N1 vaccine.

CONCLUSION. Replicating Ad4 delivered to the URT caused prolonged exposure to antigen, drove durable systemic and mucosal immunity, and proved to be a promising platform for the induction of immunity against viral surface glycoprotein targets.

TRIAL REGISTRATION. ClinicalTrials.gov NCT01443936 and NCT01806909.

FUNDING. Intramural and Extramural Research Programs of the NIAID, NIH (U19 AI109946) and the Centers of Excellence for Influenza Research and Surveillance (CEIRS), NIAID, NIH (contract HHSN272201400008C).

\section{Introduction}

Induction of a durable antibody response capable of neutralizing diverse isolates is a high priority for the development of vaccines against viruses such as HIV, severe acute respiratory syndrome coronavirus 2 (SARS-CoV-2), and influenza. In the case of HIV-1 and influenza virus, protection against infection has been demonstrated in animal models to be mediated by neutralizing antibodies

Authorship note: KM and SAM contributed equally to this work.

Conflict of interest: $M C$ is an inventor on a patent titled "Replication-competent adenovirus type 4 SAR-CoV-2 vaccines and their use (patent application no. 63/138,221). MC, JS, and JA are inventors on a patent titled "Replication-competent adenovirus type 4-HIV Env vaccines and their use" (patent application no. 63/063,810) Copyright: () 2021, American Society for Clinical Investigation.

Submitted: June 2, 2020; Accepted: January 7, 2021; Published: March 1, 2021

Reference information: J Clin Invest. 2021;131(5):e140794.

https://doi.org/10.1172/JCl140794. targeting the surface glycoproteins. In the case of influenza virus, the protection afforded by existing vaccines is not long-lived, and annual vaccination with antigens that match circulating strains as closely as possible is necessary (1). Thus, improvements in immunogenicity that lead to increases in the magnitude, durability, and breadth of the immune response are a high priority for the efforts to develop better vaccines against influenza virus. Of the available vaccine platforms for presenting these glycoproteins to the immune system, replicating vectors have several important advantages over most nonreplicating vectors (2). They can express viral surface glycoproteins such that the total dose of antigen probably exceeds those of nonreplicating vectors. In addition, viral surface glycoproteins can be expressed by the host cell in the appropriate conformation and glycosylation state. Antibodies induced by host cell-produced glycoproteins may better target virions during natural infection compared with those induced by glycoproteins 
produced in cell lines or eggs. They may also directly or indirectly stimulate B cell proliferation and differentiation through nucleic acid stimulation of TLRs in B cells or antigen-presenting cells and induce proinflammatory cytokines. In addition, they can express viral glycoproteins over a prolonged period of time, similar to other live virus infections. This feature is currently the focus of intense investigation and thought to be critical to the loading of follicular DCs in the lymph node and the induction of a durable antibody response (3). Vectors that replicate at the same mucosal sites as the target virus have particular advantages by inducing local cellular and humoral immunity, a feature that is lacking in nonreplicating, parenterally administered vaccines. Although replicating vectors offer numerous advantages, the level of immunogenicity is often modulated by the level of replication of the vector, transgene expression, preexisting immunity, and route of administration.

Among the recombinant viral vectors available for human use, replicating adenoviruses offer several important advantages. Replicating adenovirus type $4(\operatorname{Ad} 4)$ has been orally administered to more than 10 million people in the military as a vaccine against Ad4 respiratory disease and has an extraordinary safety record (4). This wild-type virus, that in natural infection replicates on the respiratory tract, is attenuated by administration in the gastrointestinal tract (GI) in the form of an enteric-coated tablet and does not cause respiratory disease. Although the seroprevalence of neutralizing antibodies is approximately $30 \%$ in adults, there are some limited data that suggest that humans are able to be reinfected with this virus $(5,6)$. Replicating recombinant adenoviral vectors have shown some promise when used to vaccinate macaques; however, a thorough evaluation of their potential immunogenicity is limited by the lack of full replication capacity in animal models (7). In one earlier trial in humans, replicating recombinant adenoviral vectors were only modestly immunogenic, probably due to attenuated replication as a result of administration to the GI tract (8).

In a more recent trial, we observed higher and more durable levels of influenza virus-specific neutralizing antibodies when a replication-competent Ad4 expressing the influenza virus $\mathrm{H} 5$ HA from A/Vietnam/1194/04 (Ad4-H5-Vtn) was administered via the upper respiratory tract (URT) versus the GI tract (9). After a single intranasal or tonsillar administration of the vaccine, we observed prolonged increases in $\mathrm{H} 5$-specific B cells, H5-specific antibody somatic hypermutation, and monoclonal antibody neutralization potency for 6 to 12 months. Thus, compared with oral administration, the platform showed considerably greater immunogenicity when administered to the URT, where its replication was only limited by attenuation due to insertion of a transgene.

In the present study, we examine the impact of replication on the induction of neutralizing antibodies in participants who received the Ad4-H5-Vtn vaccine. T cell and antibody responses, virus shedding, adenovirus-related symptoms, and induction of mucosal immunity were examined in detail. Further, we also assessed the ability to use Ad4-H5-Vtn to prime antibody responses prior to administration of inactivated or recombinant protein $\mathrm{H} 5$ vaccines. We observed that the magnitude of $\mathrm{H} 5$-specific antibody responses was associated with prolonged replication of the Ad4-H5-Vtn virus and vaccine-related adverse events (AEs) consistent with an inflammatory response. These data suggest that recombinant Ad4 vectors, when given via the URT, show considerable promise to deliver viral glycoproteins either alone or in combination with a recombinant protein or inactivated virus boost.

\section{Results}

Ad4-H5-Vtn shedding at mucosal sites. Of the 198 individuals recruited and screened for Ad4 seropositivity, 55 tested seropositive according to our selection neutralization titer criterion of an $80 \%$ inhibitory dilution $\left(\mathrm{ID}_{80}\right.$ ) above 100 . The prevalence of Ad4 seropositivity in this population $\left(\mathrm{ID}_{80}>100,28 \%\right.$ ID $_{50}>100$, $39 \%$; $_{50}>20,49 \%$ ) was consistent with prior estimates (ref. 10 and Supplemental Figure 1A; supplemental material available online with this article; https://doi.org/10.1172/JCI140794DS1). Sixty-three Ad4-seronegative participants who met the inclusion criteria and signed the informed consent were enrolled in a nonrandomized, open-label, dose-escalation study of Ad4-H5-Vtn to receive a single dose of the vaccine by an oral enteric-coated capsule $\left(10^{10}\right.$ virus particles [vp]), swabbing onto the tonsils $\left(10^{3}-10^{8}\right.$ $\mathrm{vp}$ ), or intranasal spray $\left(10^{3}-10^{8} \mathrm{vp}\right)$. In addition, Ad4-seropositive volunteers could be enrolled into a parallel exploratory vaccine arm. We observed no significant differences among the 3 vaccine groups with respect to age or sex. However, there were some differences in racial/ethnic composition, with a greater representation of White vaccinees in the oral arm (80\% versus 32\% in the tonsillar and $32 \%$ in the intranasal arms, $P=0.02)$ and Black vaccinees in the tonsillar arm (64\% versus $20 \%$ in the oral and $32 \%$ in the intranasal arms, $P=0.03$; Supplemental Table 1). Although present in relatively low numbers, we also noted a trend toward a higher percentage of Asians in the intranasal arm compared with either the oral or tonsillar arm (25\% vs. $0 \%$ and $4 \%$, respectively; $P \geq 0.05$ ). We previously reported that immunizations by these routes with a dose of $10^{4} \mathrm{vp}$ or higher resulted in seroconversion to Ad4 (9). This suggested that once infection was established, replication was sufficiently robust, even in groups that received very low doses of infectious virus (104 vp or 10 infection-forming units [IFU]), but the differences in the response to the vaccine were not discernable in our assays. To assess the duration of Ad4H5-Vtn shedding of this vaccine at mucosal sites, we measured viral DNA in nasal, tonsillar, and rectal swab extracts. The highest frequencies of viral shedding were detected in nasal swabs from the group immunized intranasally, peaking 6 days after immunization and lasting until 28 days after immunization (Figure 1A, left). High frequencies on day 1 may have been the result of residual viral DNA present from the intranasal vaccine administration. In contrast, the tonsillar group showed only modest frequencies in nasal mucosa, peaking 4 to 5 days after immunization (14.3\%). No viral shedding in nasal swabs was detected in the group immunized orally (Figure 1A, left). In tonsillar swabs, we detected viral shedding at comparable frequencies between the intranasal and tonsillar groups, peaking on day 6 (75\%). Viral shedding continued until 21 days after immunization for intranasal vaccinees, and 28 days after immunization for tonsillar vaccinees. Consistent with the frequency of nasal shedding and prior work on the orally administered wild-type vaccine, we detected no tonsillar viral shedding in the group immunized using an enteric-coated capsule (Figure 1A, middle, and ref. 8). Viral shedding was detected in rectal swabs from the intranasal (34.6\%) and tonsillar (25\%) 
A

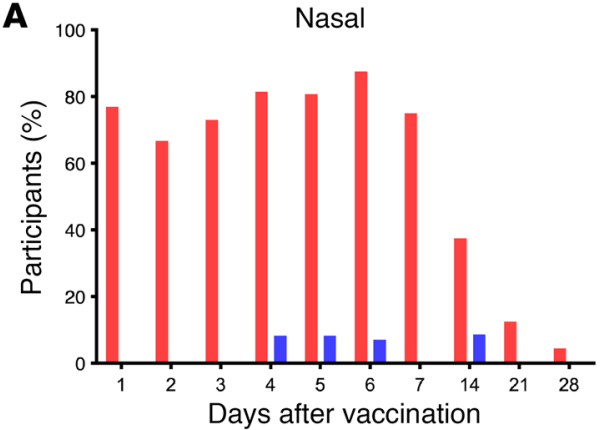

B

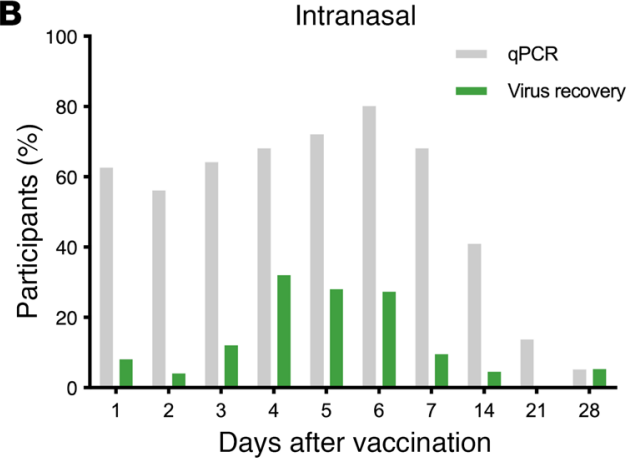

Tonsillar
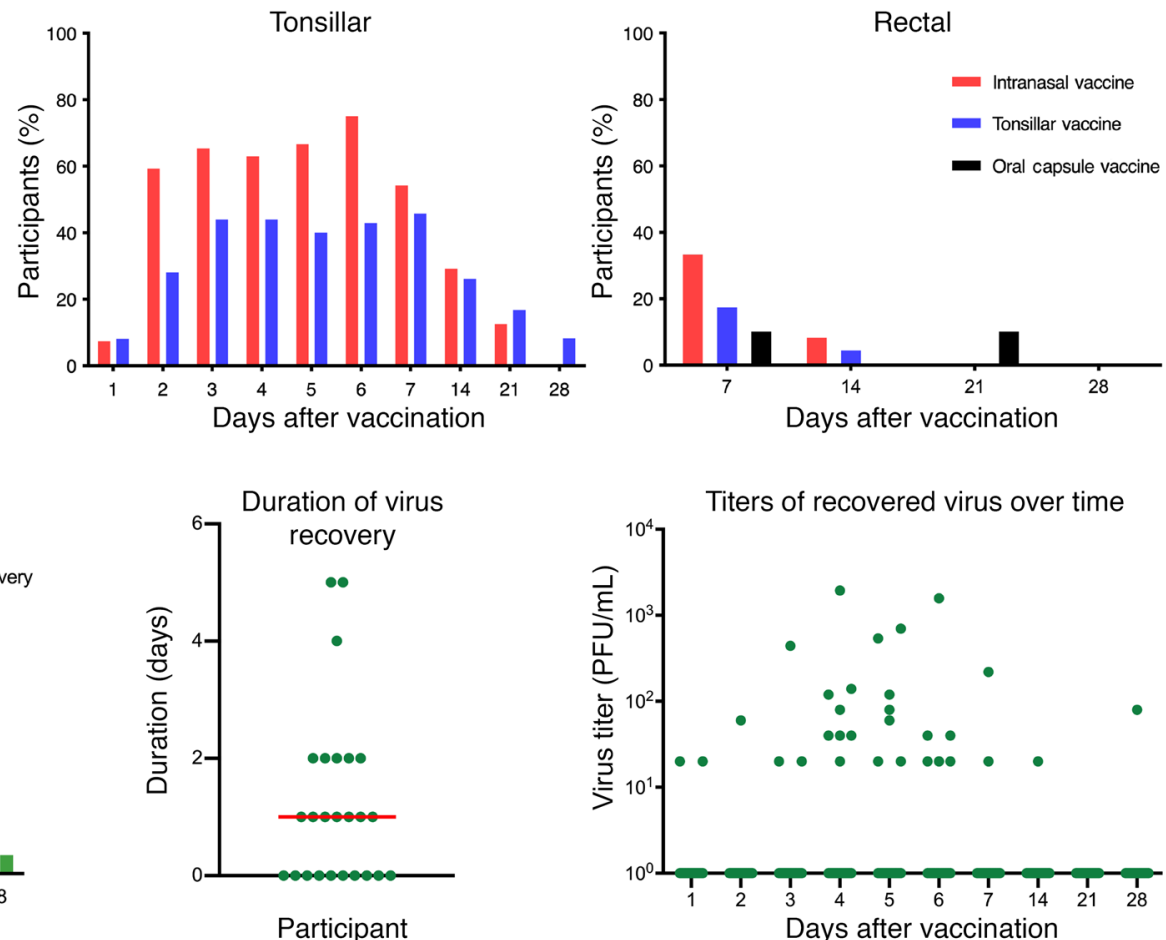

Titers of recovered virus over time

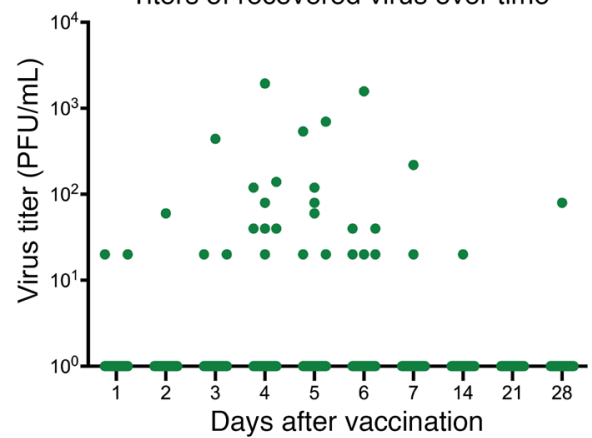

Figure 1. Ad4-H5-Vtn shedding at mucosal sites. (A) qPCR was performed on the nasal, tonsillar, and rectal swabs for the detection of viral shedding up to 4 weeks (28 days) after vaccination. Immunization is shown for intranasal (red, $n=25$ ), tonsillar (blue, $n=28$ ), and oral (black, $n=10$ ) routes. (B) Recovery of infectious virus (green) on nasal swabs was measured in comparison with qPCR (gray) in vaccinees immunized by the intranasal route (left). The distribution of days of shedding (middle) and the titers of recovered virus over time (right) are shown $(n=25)$. The red horizontal bar indicates the median value.

groups, peaking 7 days after immunization. Viral shedding was also detectable in the oral group, with a delayed peak 21 days after immunization (18\%; Figure 1A, right). The frequency of shedding detected in rectal swabs following oral administration was well below that observed in a prior trial, suggesting that there was variability in the process of sample collection (8). Consistent with our previous observation that there was no effect of dose on the development of neutralizing antibodies against the $\mathrm{H} 5$ transgene by week 8 or 26 , we observed no differences in the duration of shedding among the dose groups for tonsillar $\left(10^{4}-10^{8}\right.$ dose) or intranasal $\left(10^{4}-10^{8}\right.$ dose) administration (Figure $1 \mathrm{~A}$ and ref. 9). Taken together, these results suggested that administration of Ad4-H5-Vtn to the URT by tonsillar swab or intranasal spray resulted in robust viral replication over a prolonged period at doses of $10^{4} \mathrm{vp}$ or higher, especially at the corresponding vaccination site. This is consistent with the replication of the wild-type Ad4 virus that occurs during natural infection of the respiratory tract.

In order to assess the presence of replication-competent virus at mucosal sites, we also performed an immuno-plaque assay on cells cultured with nasal swab extracts to detect Ad4. In nasal swabs from intranasal Ad4-H5-Vtn vaccinees, we detected replication-competent virus up to 28 days after immunization (Figure 1B). The frequencies of swab extracts containing replication-competent virus, although notably lower, closely tracked with the frequencies detected by quantitative PCR (qPCR). The peak in frequency of infectious virus occurred slightly earlier (4 days after immunization) than did the peak in frequency of viral DNA detected by qPCR ( 6 days after immunization; Figure 1B, left). Only $60 \%$ of participants had shedding detectable at any time point. In addition, this shedding occurred for a brief window of time (median of 1 day; Figure 1B, middle) and was of low magnitude (<2000 IFU per swab; Figure 1B, right). This last result is offered with the caveat that quantification of virus on nasal swabs is at best semiquantitative and subject to issues of recovery from the vaccinee and from the swab. However, taken together, these results suggested that qPCR may overestimate the duration of viral shedding and that shedding of infectious virus was brief from most vaccinees and not detected in $40 \%$ of them.

Transmissibility is an important consideration in evaluating replication-competent vaccine vectors. In addition to all of the participants in the current study, we enrolled 11 intimate contacts of the vaccinees (i.e., a sexual partner of the participant) and tested them for seroconversion against Ad4. Intimate contacts and vaccinees were counseled to avoid intimate contact for 28 days after vaccination. Of those 11 intimate contacts enrolled, we did not detect evidence of seroconversion throughout a 4-week observation period after vaccination (data not shown).

Induction of H5-specific cellular and humoral responses following Ad4-H5-Vtn vaccination. To investigate the induction of $\mathrm{H} 5$-specific $\mathrm{T}$ cell responses to the Ad4-H5-Vtn vaccine, PBMCs from baseline and week 4 were stimulated with an influenza virus H5 HA peptide array and analyzed by flow cytometry (Supplemental Figure 2). The frequencies of activated, IFN- $\gamma$-secreting $\mathrm{CD} 4^{+} \mathrm{T}$ cell responses induced by the oral vaccine were low overall, but significantly higher than baseline (mean difference from week 0 to week 4, 0.016\%, $P=0.005$; Figure 2A, left). We observed 
A
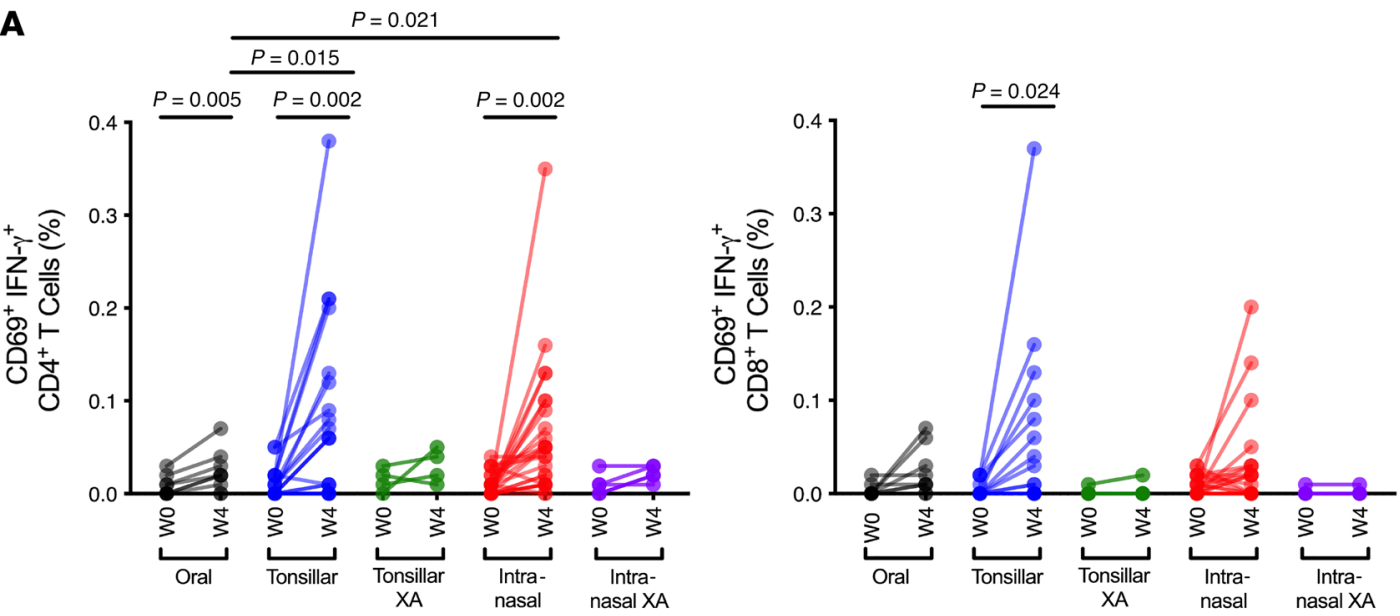

B
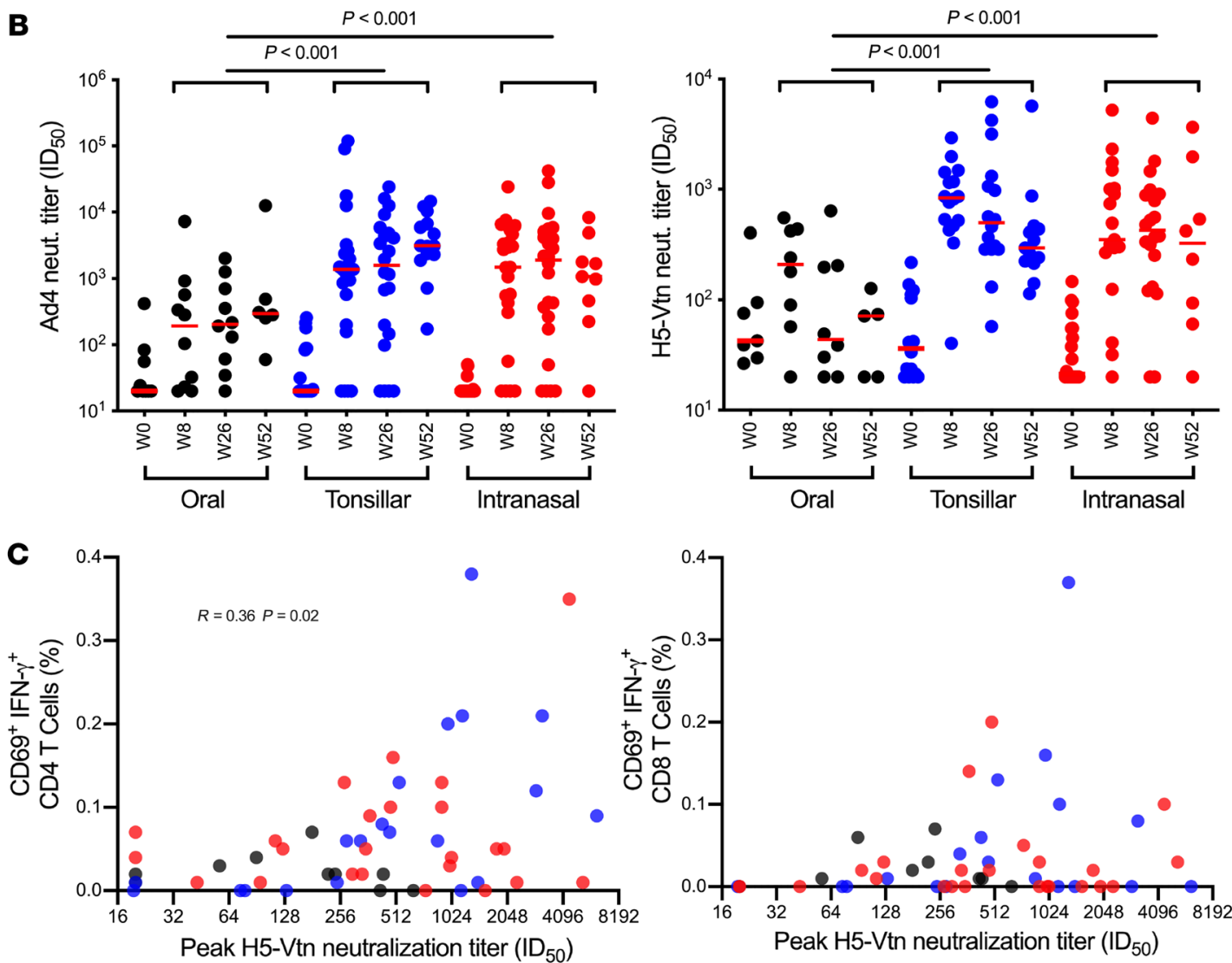

Figure 2. Induction of H5-specific cellular and humoral responses following immunization with Ad4-H5-Vtn. (A) H5-specific CD4+ and CD8 ${ }^{+} \mathrm{T}$ cell responses measured after a 6-hour stimulation with an A/Vietnam/1203/2004 (H5N1) influenza virus peptide array are shown for samples taken at baseline (week $\mathrm{O}$ [WO]) and 4 weeks (W4) after immunization with the Ad4-H5-Vtn vaccine, which had been administered as an oral capsule ( $n$ $=9)$, by direct tonsillar application $(n=20)$, or by intranasal spray $(n=25)$. In addition, responses for individuals who were Ad4 seropositive prior to vaccination are shown as separate exploratory arm (XA) subgroups (Tonsillar XA, $n=4$; Intranasal XA, $n=5$ ). Background responses to media controls have been subtracted. Paired and unpaired 2-sample $t$ tests were used for group comparisons. Only significant $P$ values are shown. (B) Ad4 (oral, $n=9$; tonsillar, $n=22$; intranasal, $n=24$ ) and $\mathrm{H} 5$ (oral, $n=7$; tonsillar, $n=16$; intranasal, $n=20$; individuals with less than a 4-fold increase in $\mathrm{Ad} 4$ and $\mathrm{H} 5$ neutralization [neut.] titer after immunization were excluded) $\mathrm{HA}$-specific neutralizing antibody titers, shown as $\mathrm{ID}_{50}$, were measured by a PVEI assay. The XA subgroups were excluded. Red horizontal bars indicate the median values. Generalized estimating equations with an autoregressive correlation structure were used to calculate $P$ values (week 0 measurements were not included), and only significant $P$ values are shown. (C) Correlation of H5-specific CD4 and CD8 responses with peak neutralization antibody titers (oral in black, $n=10$; tonsillar in blue, $n=20$; intranasal in red, $n=24$ ) were assessed by the Spearman's rank method. The XA subgroups were excluded. Only significant $P$ values are shown. In multiple-comparison calculations including all $P$ values, the FDR was estimated to be $6 \%$ using the Benjamini-Hochberg procedure, and a fixed $P$ value significance threshold was set at 0.05 . 
A

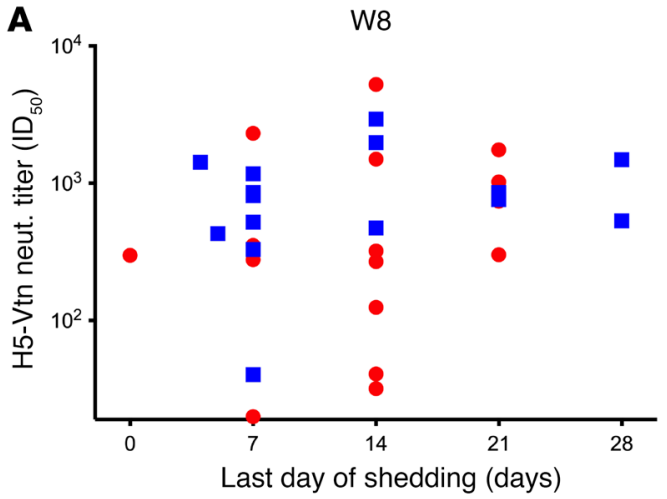

B

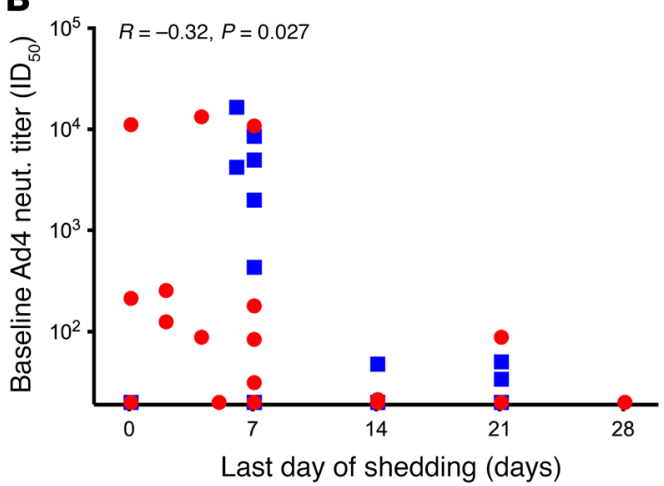

W26
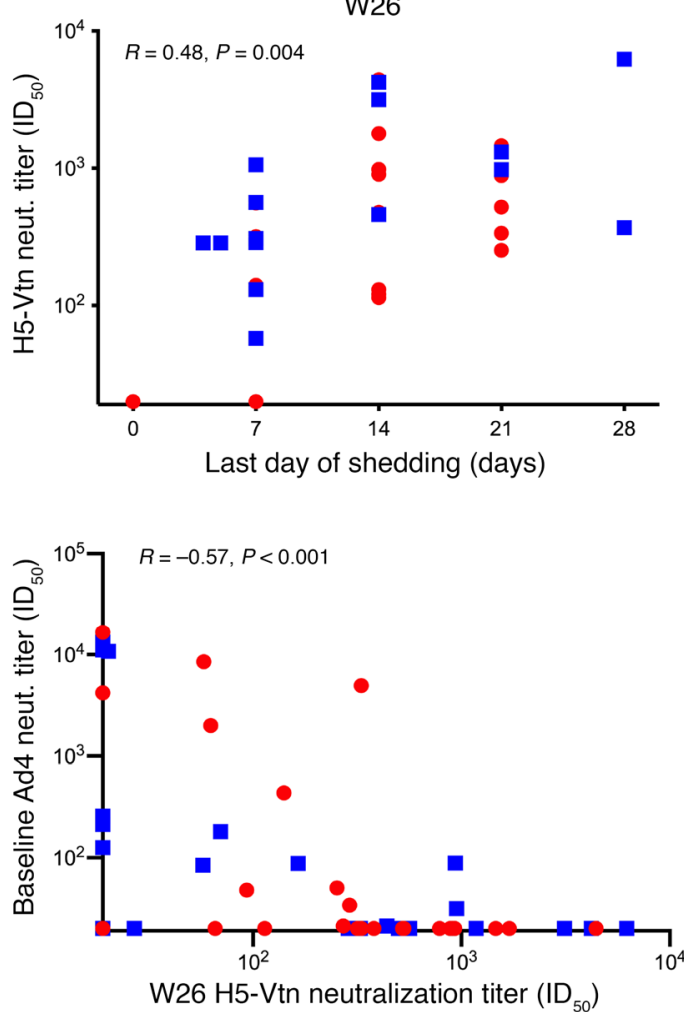
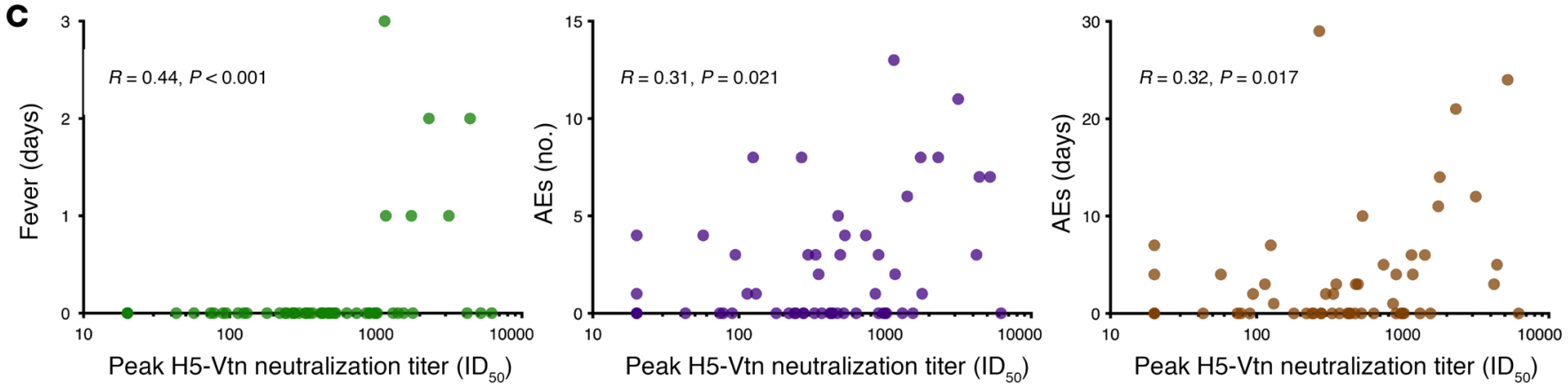

Figure 3. Clinical correlates of anti-H5-neutralizing antibody titers. (A) The correlation between H5-neutralizing antibody titers 8 weeks (left, $n=32$ ) and 26 weeks (right, $n=33$ ) after vaccination and the last day of viral shedding detected was assessed for participants from the tonsillar (blue squares) and intranasal (red circles) groups who had a 4-fold increase in Ad4-neutralizing antibody titers after vaccination. Significance was determined by Spearman's rank method. Only significant $P$ values are shown. (B) The correlation between Ad4-neutralizing antibody titers at baseline and the last day of detectable viral shedding (left, $n=48$ ) and peak H5-Vtn neutralization titers (right, $n=49$ ) was assessed by the Spearman's rank method. Symbols are as in $\mathbf{A}$. Vaccinees who did not seroconvert to Ad4 are included for the purpose of this analysis. (C) The correlations between peak $\mathrm{H} 5$-neutralizing antibody titers after vaccination and the duration of related fever (days, left), the total number of participants with related AEs (middle), and the duration of related AEs (days, right) was assessed among vaccine recipients in the oral, tonsillar, and intranasal arms ( $n=56$; participants with matching AEs and H5-neutralizing antibody titers are shown). The Spearman's rank method was used to determine significance.

greater differences between baseline and week 4 responses in the tonsillar and intranasal arms $(0.074 \%, P=0.002$ and $0.054 \%$, $P=0.002$, respectively; Figure $2 \mathrm{~A}$, left), confirming higher replication and immunogenicity of this vaccine when delivered to the URT. We found that significant increases above baseline were not induced in the small numbers of vaccine recipients in the exploratory arms who had been immunized via the tonsillar or intranasal routes (Figure 2A, left). Trends for the induction of H5-specific $\mathrm{CD}^{+} \mathrm{T}$ cell responses were similar to those for $\mathrm{CD} 4^{+} \mathrm{T}$ cell responses, although significant increases from week 0 to week 4 occurred only in the tonsillar arm (mean difference $0.046 \%, P=$
0.024; Figure 2A, right). Vaccine-induced $\mathrm{H} 5$-specific $\mathrm{CD}^{+}{ }^{+} \mathrm{T}$ cell frequencies tended to be higher than $\mathrm{CD}^{+} \mathrm{T}$ cell frequencies at week 4 in all vaccine subgroups, but this difference achieved statistical significance only in the intranasal arm (mean difference $0.037 \%, P=0.046$; Figure 2A, left and right panels).

Ad4-specific neutralizing antibody titers increased in all 3 groups 8 weeks after immunization compared with baseline titers (oral: $P=0.032$, tonsillar: $P<0.001$, intranasal: $P<0.001$; Figure $2 \mathrm{~B}$, left). Groups receiving the vaccine via the URT showed significantly higher overall post-vaccination Ad4-specific neutralizing antibody titers compared with the group receiving the vaccine capsule orally 
Table 1. AEs for all cohorts with possible, probable, or definite relatedness to Ad4-H5-Vtn vaccination

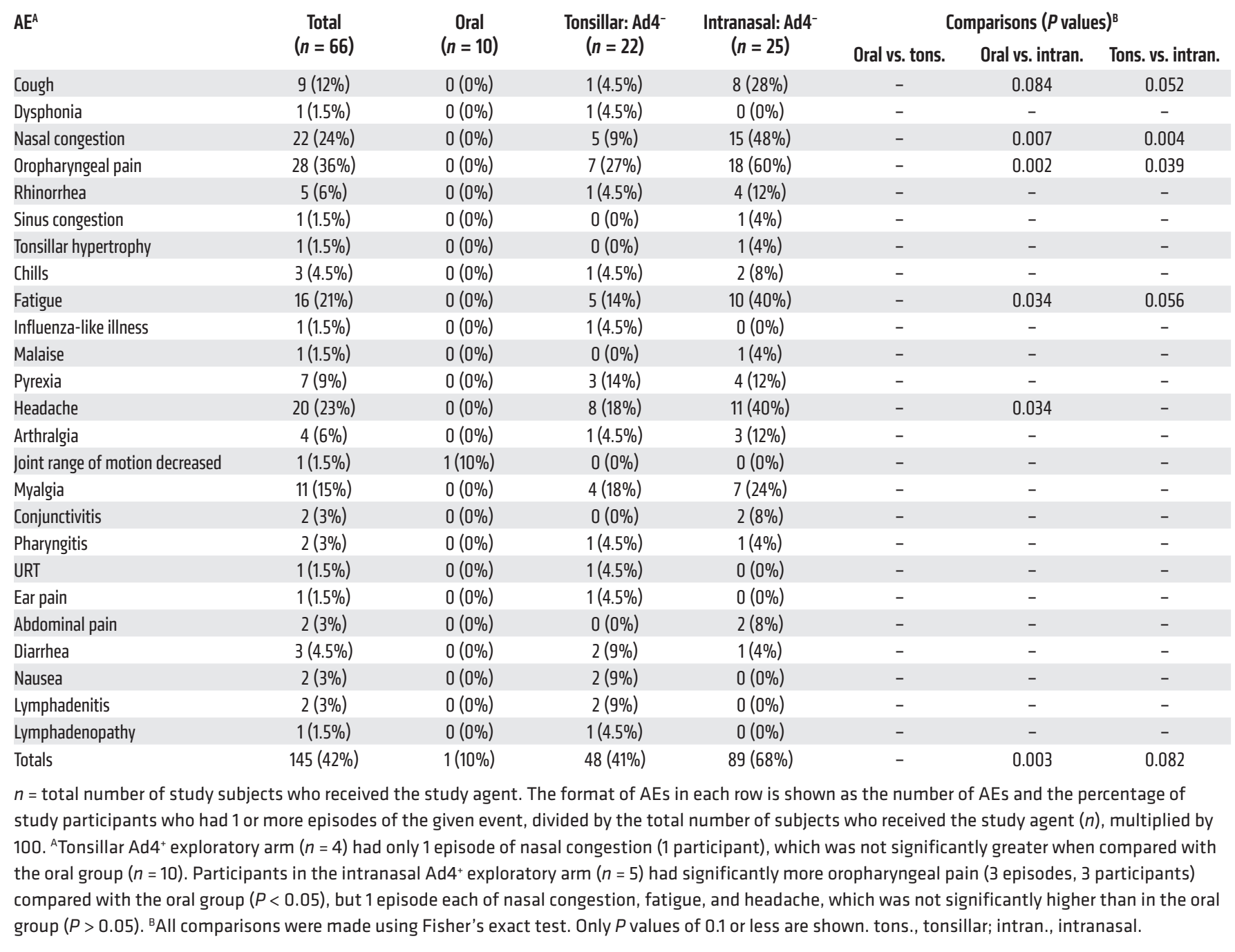

(tonsillar: $P<0.001$, intranasal: $P<0.001)$. In contrast, we found no significant difference between the groups receiving the vaccine via the URT ( $P=0.561)$. Significant increases in H5-specific neutralizing antibodies, measured in a pseudotyped virus inhibition assay (PVEI), were also detected 8 weeks after immunization (oral: $P=0.008$, tonsillar: $P<0.001$, intranasal: $P<0.001)$ compared with baseline. Similar to Ad4-specific neutralizing antibodies, we found that a greater magnitude of $\mathrm{H} 5$-specific neutralizing antibodies were induced in the groups receiving the vaccine via the URT (tonsillar: $P<0.001$, intranasal: $P<0.001)$ compared with the group receiving the vaccine capsule orally. There was no significant difference in H5-specific neutralizing antibody titers between the groups receiving the vaccine via the intranasal or tonsillar route $(P=0.112$; Figure $2 \mathrm{~B}$, right). In addition, week-26 $\mathrm{H} 5$-specific neutralizing antibody titers were comparable between Black and White vaccinees in the tonsillar $\operatorname{arm}(P=0.757)$ and between Black, White, and Asian vaccinees in the intranasal arm $(P>0.05$ for all comparisons; data not shown). Neutralization titers remained significantly different between the oral and tonsillar/intranasal arms $(P=0.047)$ and similar between the tonsillar and intranasal arms $(P=0.717)$ when comparisons were limited to White vaccinees. We observed the same trends in comparisons of peak $\mathrm{H} 5$ neutralization titers. Overall, these findings suggested that disparities in racial/ethnic composition among the arms were unlikely to account for the increased neutralization capacity induced by vaccines delivered to the URT. The frequencies of $\mathrm{H} 5$-specific $\mathrm{CD}^{+} \mathrm{T}$ cells showed a modest but significant correlation with the peak serum neutralizing antibody titers $(R=0.36, P=$ 0.02 ) when all participants were grouped together (Figure $2 \mathrm{C}$, left). However, when participants were grouped by immunization routes, this correlation appeared to be strongest in the tonsillar group $(R=$ $0.69, P \leq 0.001)$. In contrast, the frequencies of $\mathrm{H} 5$-specific $\mathrm{CD} 8^{+} \mathrm{T}$ cells did not correlate with neutralization capacity (Figure $2 \mathrm{C}$, right).

In summary, these results indicate that greater $\mathrm{H} 5$-specific $\mathrm{T}$ cell and neutralizing antibody responses were induced with URT administration. In addition, this vaccine platform appeared to be a poor overall inducer of $\mathrm{H} 5$-specific $\mathrm{T}$ cell responses but induced moderate levels of H5-specific antibody with considerable durability, probably through the induction of long-lived plasma cells. 
A

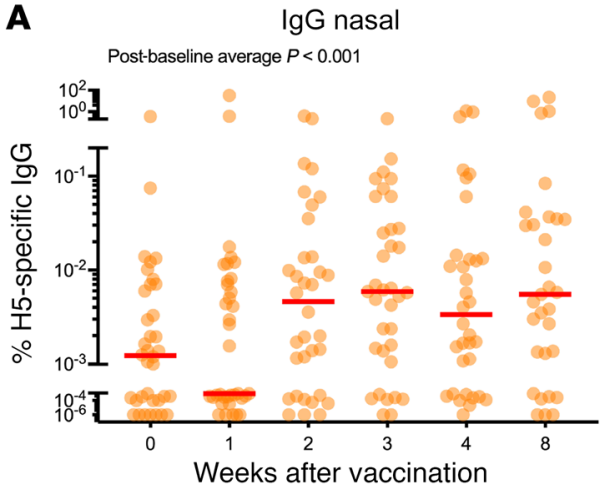

IgA nasal

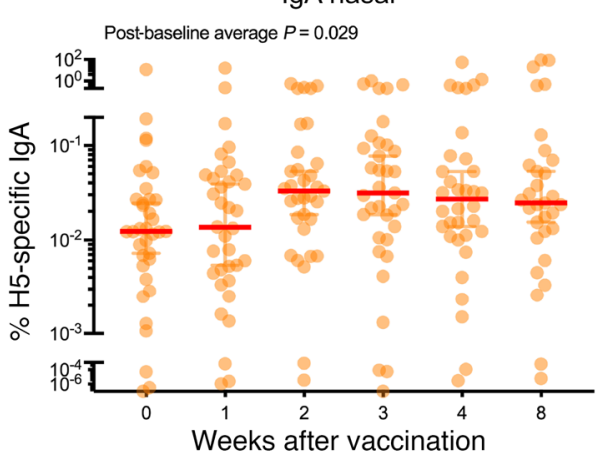

IgG rectal

Post-baseline average $P<0.001$

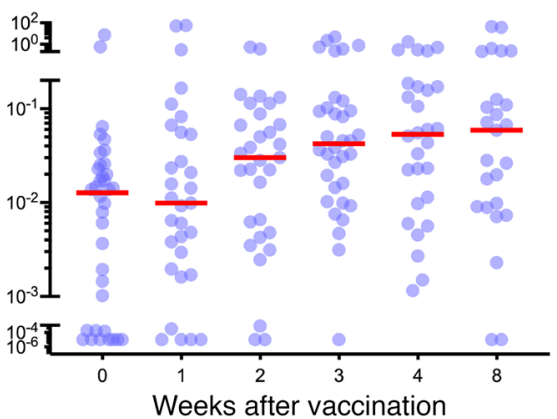

$\lg$ A rectal

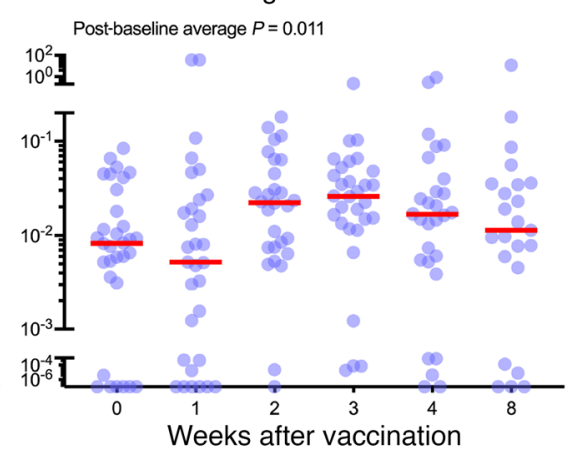

lgG cervical

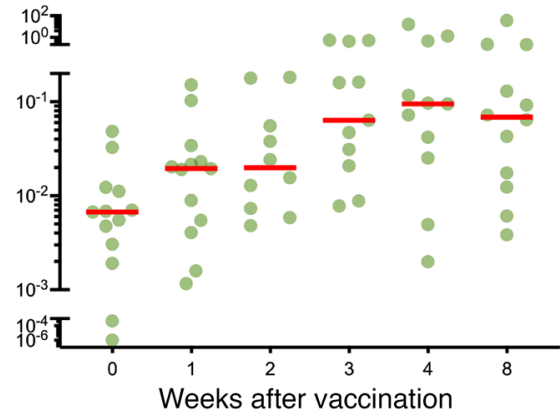

$\lg$ A cervical

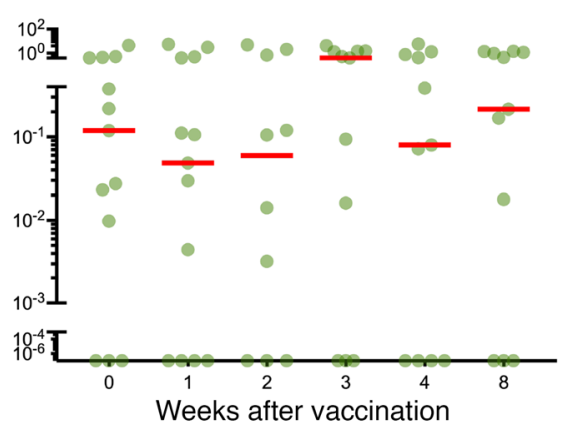

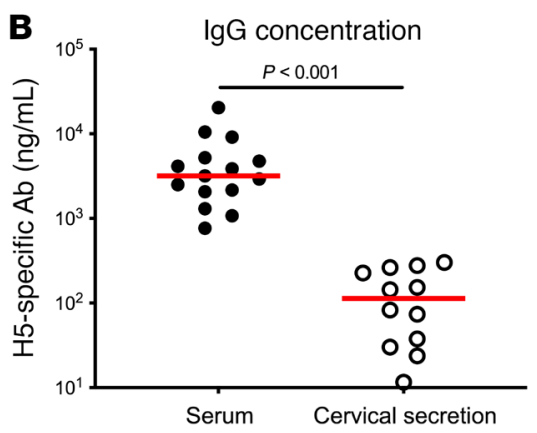

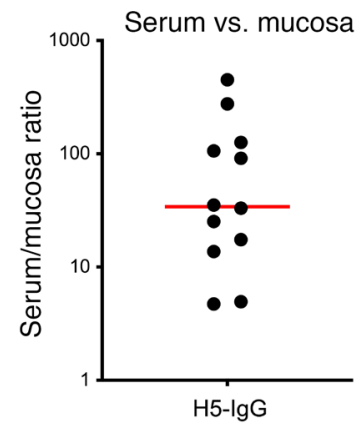

Figure 4. Induction of H5-specific antibodies at mucosal sites. (A) The proportions of H5 HA-specific IgG ( $n=14-37)$ and IgA $(n=13-37)$ in nasal (orange), rectal (blue), and cervical (green) secretions were calculated against total IgG and IgA. All available samples from intranasal and tonsillar groups were assessed. Participants who were seropositive for Ad4 at baseline were excluded. Red horizontal bars indicate median values. Generalized estimating equations with an autoregressive correlation structure were used to calculate $P$ values (week 0 measurements were not included), and only significant $P$ values are shown. (B) Shown are serum $(n=15)$ and cervical secretion $(n=12)$ concentrations of H5-specific IgC 4 weeks after vaccination and the ratio of $\mathrm{H} 5$-specific antibodies in serum versus cervical secretions $(n=12)$. A paired $t$ test was used to calculate $P$ values. Red horizontal bars indicate median values.
Clinical correlates of anti-H5-neutralizing antibody titers. One potential advantage of replication-competent vaccine platforms is that they can induce a higher or more durable immune response by presenting antigens for a prolonged period of time. To investigate this, we assessed the correlation between the duration of Ad4-H5-Vtn viral shedding and antigen-specific serum neutralization titers. Although H5-specific neutralizing antibody titers at week 8 were not significantly correlated with the duration of viral shedding $(P=0.223$; Figure $3 \mathrm{~A}$, left $)$, they were modestly correlated at week 26 ( $R=0.48, P=0.004$; Figure $3 \mathrm{~A}$, right). This suggested that prolonged viral shedding has an impact on the magnitude or durability of the $\mathrm{H} 5$-specific antibody response but requires time to manifest.

To assess the potential effect of preexisting immunity on Ad4$\mathrm{H} 5-\mathrm{Vtn}$ replication and shedding, we performed a correlation analysis of pre-vaccination Ad4-specific antibody titers and the duration of Ad4-H5-Vtn shedding. For this analysis, we pooled data from baseline Ad4-seronegative participants with those that were Ad4 seropositive in the exploratory arm. We found that pre- existing Ad4-specific neutralizing antibody levels were modestly negatively correlated with the duration of Ad4-H5-Vtn shedding $(R=-0.32, P=0.027$; Figure $3 \mathrm{~B}$, left). Regardless of baseline serostatus, nearly all participants were able to be infected by URT administration when receiving $10^{4}$ vp or more. Only 7 participants were not infected via the URT, and they had baseline serum neutralizing titers of $20,20,214,257,13,346,11,113$, and 10,815. However, none of the participants with a baseline Ad4 neutralization titer above $100 \mathrm{ID}_{80}$ shed the virus for more than 7 days. Further, we noted a significant inverse correlation of the baseline Ad4 neutralization titer and the week-26 response to $\mathrm{H} 5(R=-0.57$ $P \leq 0.001$; Figure 3B, right). Taken together, these data suggested that, although most participants were able to be infected via the URT, preexisting immunity limited the period of virus shedding. This limitation on replication of the virus likely affected the magnitude of the immune response to $\mathrm{H} 5$.

Another advantage of replication-competent vaccine platforms is that they can induce proinflammatory responses similar to those of natural viral infection. These inflammatory respons- 
A

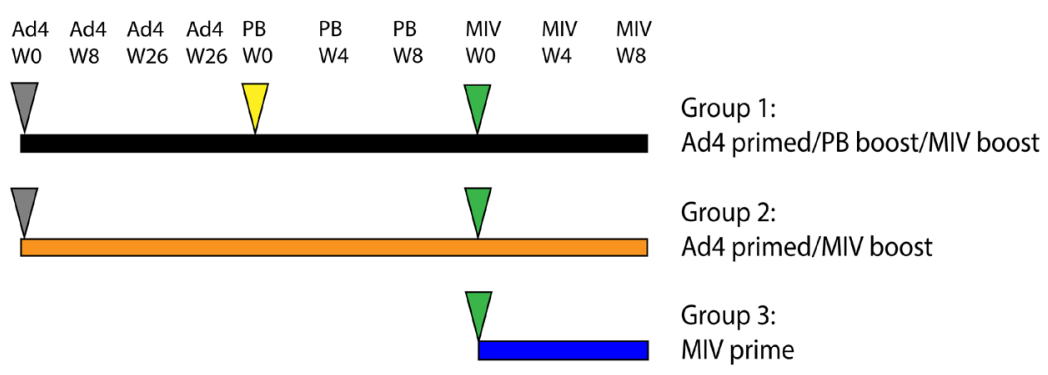

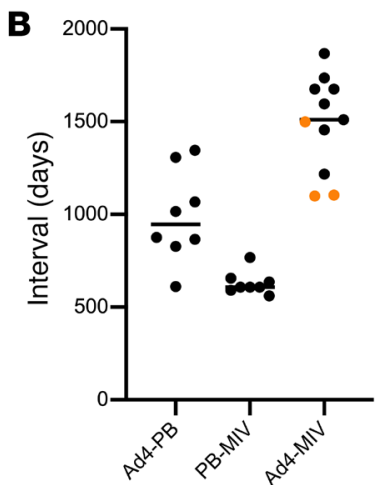

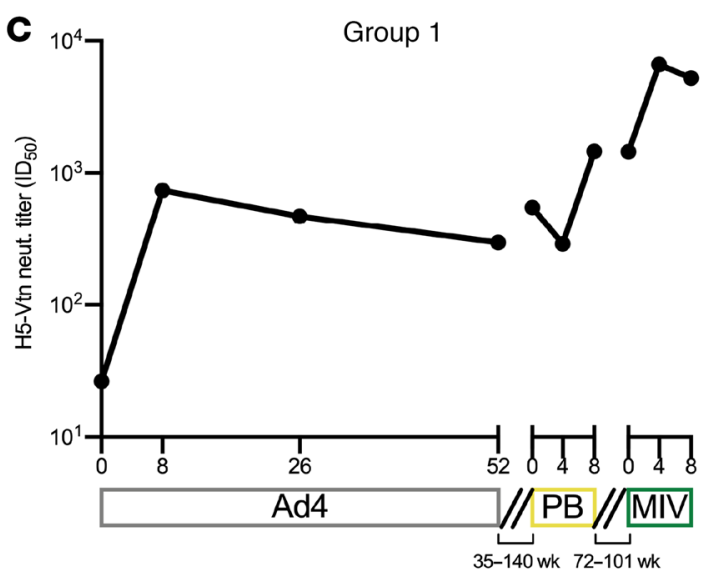

D

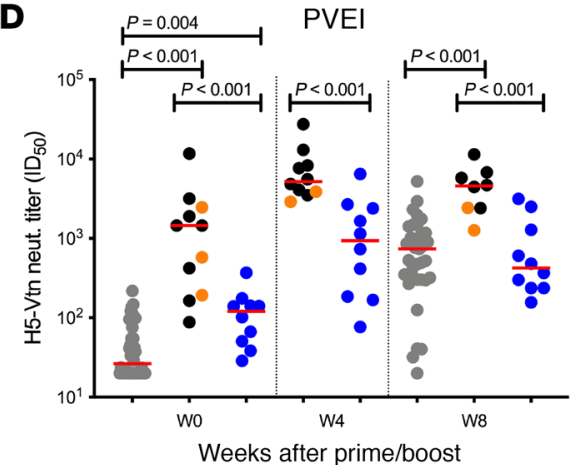

E

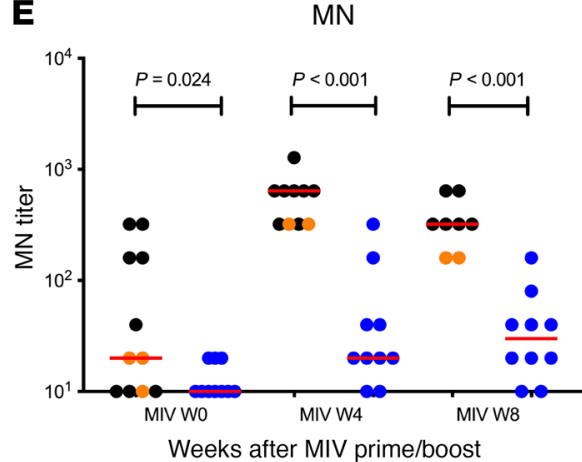

Group 2
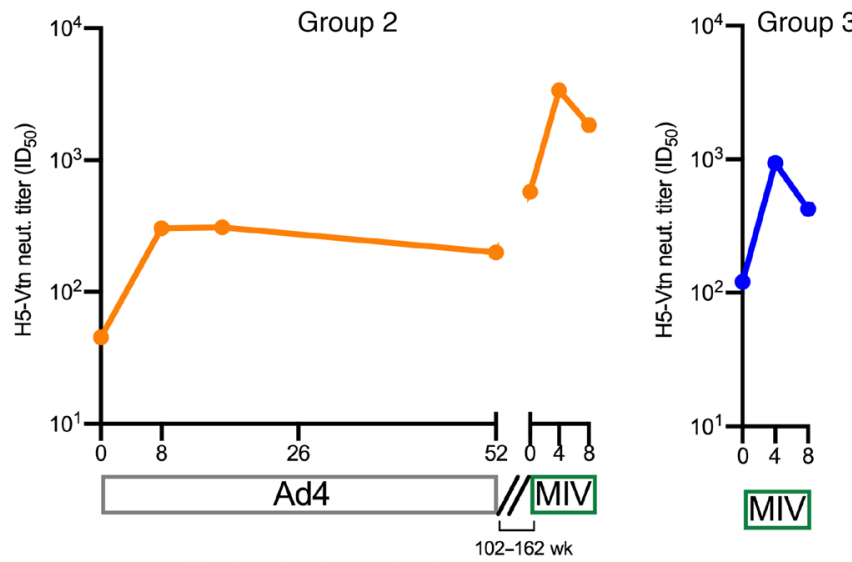

Figure 5. Neutralization antibody titers after a MIV prime/boost. (A) Immunization schedule. Ad4 (gray triangles), PanBlok (PB) (yellow triangle), and MIV (green triangles) immunizations are shown. (B) Interval between each immunization. Black circles indicate the participants in group 1, and orange circles indicate the participants in group $2(n=3)$. Participants with less than a 4 -fold increase in Ad4 neutralization titers after immunization were excluded. (C) Longitudinal changes in the median $\mathrm{H} 5 \mathrm{HA}$-specific neutralization antibody titers, shown as the median $\mathrm{ID}_{50}$ in serum from participants in group 1 ( $n$ $=8)$, group $2(n=3)$, and group $3(n=10)$, were measured by the PVEl assay. The same exclusion criteria as in B were applied. The intervals between each immunization are shown as disconnected lines between time points. (D) Post-vaccination MIV prime/boost H5 HA-specific neutralizing antibody titers in serum from participants group 1 (black, $n=8$ ) group 2 (orange, $n=3$ ), and group 3 (blue, $n=10$ ) were measured by the PVEl assay. The time points for before and 8 weeks after Ad4 priming (intranasal and tonsillar groups combined are shown in gray; $n=36$ ) are also included for comparison. The same exclusion criteria as in $\mathbf{B}$ were applied. Two-sample $t$ tests were used to calculate $P$ values. Red horizontal bars indicate median values. (E) Post-vaccination MIV prime/boost H5 HA-specific neutralizing antibody titers in serum were measured by MN and HAl assays (group 1, $n=8$; group 2, $n=3$; group $3, n=$ 10). The same exclusion criteria as in B were applied. Red horizontal bars indicate median values. Two-sample $t$ tests were used to calculate $P$ values. Only significant $P$ values are shown. In multiple-comparison calculations including all $P$ values, the FDR was estimated to be $3 \%$ using the Benjamini-Hochberg procedure, and a fixed $P$ value significance threshold was set at 0.05 .

es may be manifested in vaccine-related symptoms. Thus, we assessed the incidence of related respiratory symptoms and fever against induction of $\mathrm{H} 5$-specific neutralization antibody responses. Attribution of possible, probable, or definite relatedness of AEs to vaccination was based on characteristics of being a known clinical feature of acute adenovirus infection and a temporal association of 2 to 14 days between vaccine administration and $\mathrm{AE}$ occurrence. Overall, this vaccine was associated with lowgrade ( $\leq 2)$ upper respiratory symptoms (Table 1 ), which occurred in more participants in the intranasal arm relative to the oral arm 
$(P=0.003)$. There was also a trend toward higher numbers of total AEs in the intranasal arm compared with the tonsillar arm $(P=0.082)$, which achieved statistical significance for nasal congestion $(P=0.004)$ and oropharyngeal pain $(P=0.039$; Table 1). Fever duration significantly correlated with peak $\mathrm{H} 5$-specific neutralizing antibody activity after vaccination (weeks $8-52, R=$ $0.44, P<0.001$; Figure 3C, left). The total number of related AEs and their duration (number of days) were weakly, but significantly, correlated with peak H5-specific neutralizing antibody titers after vaccination $(R=0.31, P=0.021$ and $R=0.32, P=0.016$, respectively; Figure $3 \mathrm{C}$, middle and right). Since nasal congestion and oropharyngeal pain were found to occur more frequently in the participants who received the vaccine intranasally, the frequency and duration of these AEs were also correlated with neutralizing activity. Weak but significant correlations were found between peak $\mathrm{H} 5$-specific neutralizing activity after vaccination and the number of nasal congestion events and their duration ( $R=0.27, P=0.044$ and $R=0.27, P=0.046$, respectively) and the number of oropharyngeal pain events and their duration $(R$ $=0.32, P=0.017$ and $R=0.35, P=0.009$, respectively; Supplemental Figure $3 C$ ). These results suggested that manifestations of significant symptoms in Ad4-H5-Vtn vaccine recipients, although not required, were associated with a more potent neutralizing antibody response to $\mathrm{H} 5 \mathrm{HA}$.

In order to determine whether these AEs were associated with proinflammatory cytokine levels that had been induced by vaccination, the SOMAscan assay was used to measure the levels of IL- $1 \alpha$, IL- $1 \beta$, IL- 6 , TNF- $\alpha$, and TNF- $\beta$ in serum post immunization. Although there were increases in TNF- $\beta$ in the serum of 2 participants with fever, this did not achieve statistical significance compared with those without fever. Overall, there were no significant differences in these cytokine levels when compared between participants with or without AEs (Supplemental Figure $4 \mathrm{~A}$ ) and between those with or without fever (Supplemental Figure $4 \mathrm{~B}$ ). These results indicated that this vaccine strategy induced detectable levels of proinflammatory cytokines, but these were not extremely high overall, nor were they enriched in those who experienced AEs.

One additional concern for respiratory virus vaccines is the potential to induce vaccine-associated enhanced disease (VAED) observed upon subsequent infection with the target virus (11). During the development of inactivated measles and respiratory syncytial virus (RSV) vaccines, VAED caused severe disease and even death in some immunized children upon natural infection (12). Based on animal models, this phenomenon is thought to be caused by the combination of induction of a skewed $\mathrm{T}$ cell response and a nonprotective $\mathrm{CD}^{+} \mathrm{T}$ cell and humoral response. Upon infection, there is an exaggerated $\mathrm{CD} 4^{+} \mathrm{T}$ cell response, primarily Th2 in some experimental animals, in the face of an excess of antigen caused by the nonprotective immune response. For this reason, the measurement of Th1- and Th2-related cytokines following vaccination is of interest. In an analysis of serum cytokines by the SOMAscan assay, we observed a skewing toward a Th1 response with a detectable rise in IFN- $\gamma$ and IL-2. We noted a concomitant drop in the Th2 cytokines IL-4, IL-5, IL-10, and IL-13. Thus, immunization with this replicating vector was temporally associated with a distinct Th1 cytokine response.
Induction of $\mathrm{H} 5 \mathrm{HA}$-specific antibodies at mucosal sites. The induction of neutralizing antibodies at mucosal sites, especially in the URT, may contribute to protection against respiratory pathogens (13). To assess the induction of H5-specific IgG and IgA at mucosal sites over time, nasal and rectal swabs and cervical secretions from vaccinees were examined at various time points. We detected a significant increase in $\mathrm{H} 5$-specific $\operatorname{IgG}$ and $\operatorname{IgA}$ in nasal and rectal swabs after vaccination (post-baseline average; nasal IgG: $P<0.001$, nasal IgA: $P=0.029$, rectal IgG: $P=0.001$, rectal IgA: $P=0.011$; Figure $4 \mathrm{~A})$. Although we did not detect an increase of $\mathrm{H} 5$-specific IgA in cervical secretions over time after vaccination in the small numbers of female participants $(P=$ $0.258)$, we observed a trend toward an increase in H5-specific IgG $(P=0.079)$. The high baseline H5-specific IgG and IgA is consistent with published results and may represent background caused by nonspecific binding to $\mathrm{H} 5 \mathrm{HA}$, or binding of cross-reactive anti-HA stem antibodies induced by previous exposure to other strains of influenza virus. To determine whether the existence of baseline Ad4-neutralizing antibody activity in serum could potentially affect vaccine-induced $\mathrm{H} 5$-specific antibody levels at mucosal sites, we made comparisons of H5-specific binding antibody titers in mucosa between participants with or without baseline Ad4-neutralizing antibody activity. We found no significant difference in vaccine-induced H5-specific IgG and IgA between the groups with or without baseline Ad4-neutralizing antibody activity at any of the time points (Supplemental Figure 6). Altogether, these results indicated that Ad4-H5-Vtn was capable of inducing $\mathrm{H} 5$-specific IgG and IgA antibodies at mucosal sites.

Use of the soft cervical cup permitted a direct quantitative measurement of the concentration of H5-specific antibody in cervical mucosal secretions, without the use of swabs or dilution common to other techniques. Nasal wash, swab, or wick procedures are thought to be only semiquantitative and subject to discrepancies in the amount of secretions and recovery of lavage fluid. Although influenza virus infection does not occur by the genital route, understanding the relative amounts of virus-specific antibodies in secretions is of interest for the use of this platform as a vaccine for viruses that may be spread via sexual transmission. To assess the degree of H5-specific antibody induction at mucosal sites relative to serum, we measured the levels of serum H5-specific IgG in vaccinees 4 weeks after vaccination by the same technique. H5-specific IgG was more abundant in serum compared with cervical secretions $(P<0.001$; Figure $4 \mathrm{~B}$, left). The median fold difference between $\mathrm{H} 5$-specific antibodies in serum and cervical secretions 4 weeks after immunization was 34, ranging from 5 to 450 . This result indicated that H5-specific antibodies were less abundant in mucosal secretions compared with serum, and there was a large splay in the amount of IgG in the mucosa relative to serum among vaccinees (Figure $4 \mathrm{~B}$, right). In summary, the above results indicated that single-dose URT immunization with Ad4-H5-Vtn could induce H5-specific IgG and IgA at distal mucosal sites.

Ad4-H5-Vtn as a priming vaccine. The degree to which the neutralizing antibody responses observed here might translate to protection against infection is unclear. Although a single-dose URT administration of Ad4-H5-Vtn induced a durable H5-specific serum neutralizing antibody response, it was possible that this response was not sufficiently protective and would require boost- 
ing with another modality to observe stronger responses. In prior work, it has been observed that following vaccination with replicating or nonreplicating vectors, increases in serum neutralizing antibodies can be induced with a protein vaccine boost (14-16). To examine this possibility, the participants who had received Ad4-H5-Vtn were divided into 2 groups: group 1 was boosted with monovalent pandemic recombinant HA influenza virus vaccine (PanBlok, Sanofi Pasteur) and group 2 was not. Participants in both groups then received a boost with the inactivated monovalent influenza virus vaccine (MIV) H5N1(A/Vietnam/1203/2004) (Sanofi Pasteur). In addition, a group of participants not previously immunized with $\mathrm{H} 5$ antigen were recruited to serve as controls and received a single dose of MIV (group 3). A schematic of the immunization schedule (Figure 5A) and the interval between each vaccination (Figure $5 \mathrm{~B}$ ) are shown. Longitudinal changes in the median serum H5-specific neutralizing antibody titers in each group were assessed after each immunization time point by PVEI assay. Of note, we detected little change in the neutralization titers in participants from the peak value 3 to 5 years earlier, indicating that the response to $\mathrm{Ad} 4-\mathrm{H} 5-\mathrm{Vtn}$ was very durable. The serum H5-specific neutralizing antibody titers increased after each immunization with PanBlok or MIV (Figure 5C). The serum from the control group (group 3) showed a significant increase in $\mathrm{H} 5$-specific neutralizing antibody titers, peaking at 4 weeks (median $\mathrm{ID}_{50}=939$ ) and maintaining a modest level 8 weeks after immunization (median $\mathrm{ID}_{50}=424$; Figure 5, C and D). The Ad4primed groups (groups 1 and 2 ) showed increases in neutralization potency, peaking at 4 weeks (median $\mathrm{ID}_{50}=5197$ ) and maintaining a similar level 8 weeks (median $\mathrm{ID}_{50}=1852$ ) after immunization. Neutralizing antibody titers were significantly higher in the Ad4-primed groups compared with the control group at both 4 weeks $\left(\mathrm{ID}_{50}: P<0.001\right)$ and 8 weeks $\left(\mathrm{ID}_{50}: P<0.001\right)$ after immunization. Note that the background neutralization at week 0 for the control group (median $\mathrm{ID}_{50}=120$ ) was potentially due to cross-reactive antibodies induced by previous exposure to other strains of influenza, whereas $\mathrm{H} 5$ neutralization in the Ad4-primed group (median $\mathrm{ID}_{50}=1449$ ) may have been from the combination of H5-specific neutralization induced by immunization and/or antibodies induced by previous infection. The baseline levels of neutralization potencies prior to boosting were statistically different between the control and Ad4-primed groups by a median of 12 -fold ( $\left.\mathrm{ID}_{50}: P<0.001\right)$. Although there was no statistically significant difference in peak $\mathrm{H} 5$-specific neutralizing antibody titers 8 weeks after Ad4 priming, at the same time point from the MIVprimed control group, the difference was significant against the MIV-boosted groups ( $\mathrm{ID}_{50}: P<0.001$; Figure 5D).

It is thought that the use of the PVEI potentially yields higher neutralization values than plaque reduction assays such as microneutralization (MN) or HA inhibition (HAI) assays, although results from these 3 assays tend to correlate (17). To better understand the potency of serum antibodies in other assays, we conducted MN and HAI assays on these sera. In the MN assay, neutralizing activity in sera from the control group was detectable at both 4 weeks (median MN titer $=20$ ) and 8 weeks (median MN titer $=30$ ) after immunization. The $\mathrm{MN}$ assay showed a similar pattern of increased neutralization potency in sera from the Ad4-primed group that peaked at 4 weeks (median MN titer $=640$ ) and was maintained at similar levels at 8 weeks (median MN titer $=320$ ). The difference in neutralization potency between the control group and the Ad4primed group was significant at both $4(P<0.001)$ and $8(P<0.001)$ weeks after immunization, similar to the magnitude observed in the PVEI assay (Figure 5E, left). Based on the HAI assay, most of the serum from vaccinees in the control group showed no neutralization against $\mathrm{H} 5 \mathrm{~N} 1$ at baseline or following immunization, except for serum from 1 participant, who had a moderate HAI titer $4($ HAI titer $=40)$ and $8($ HAI titer $=20)$ weeks after vaccination. Sera from the Ad4-primed group showed increased neutralization activity, peaking by week 4 after vaccination (median HAI titer = 80), and the majority of individuals maintained an HAI titer above 50 (median HAI titer $=80$ ) at the 8 -week post-immunization point. The difference in neutralization potency between the control group and the Ad4-primed group was significant both 4 weeks $(P<$ $0.001)$ and 8 weeks $(P<0.001)$ weeks after immunization (Figure $5 \mathrm{E}$, right). There was no notable difference in neutralizing antibody titers between the Ad4-primed group boosted with the inactivated MIV alone and the group boosted with a combination of the MIV and the monovalent pandemic recombinant HA influenza virus vaccine, as measured by all 3 neutralization assays. In summary, these results suggested that Ad4-H5-Vtn can serve as an effective priming agent in combination with commercially available $\mathrm{H} 5 \mathrm{vac}-$ cines. This combination induced more potent serum neutralizing antibodies compared with the MIV alone. In addition, although not directly assessed here, the response was much more durable compared with the response to the MIV alone, according to previously published results (18).

\section{Discussion}

The results of this study demonstrate the utility of the replicating Ad4 platform and provide some indications regarding the mechanisms by which Ad4 might induce a durable neutralizing antibody response. Replicating Ad4-H5-Vtn, when applied to the URT, induced a higher and more durable level of neutralizing antibodies compared with the orally administered vaccine. The contribution of prolonged replication or inflammation to the response is underscored by the association we observed between the last day of viral shedding or AEs and response magnitude. The vaccine was also able to dramatically increase responses to the licensed H5-MIV vaccine and induce mucosal immunity at multiple sites. Taken together with the previously observed stimulation of prolonged evolution of the $\mathrm{B}$ cell response following vaccination, the Ad4 platform shows considerable promise for use in vaccine regimens designed to stimulate B cell responses to viral surface glycoproteins. The prolonged expression of viral proteins by a replicating vector demonstrated here represents a favorable characteristic and an area of intense current interest. Prolonged exposure to antigen is thought to provide a response of higher magnitude and durability through the retention of antigen on lymph node DCs by immune complexes. In a recent study, delivery of HIV Env trimers by an osmotic pump stimulated higher levels of neutralizing antibodies in macaques compared with the same dose delivered by bolus (3). In our study, there was an association between the duration of replication and the magnitude of the antibody response to $\mathrm{H} 5$ that was likely related in part to the very large total antigen dose delivered by a replicating vector. This feature, coupled with 
the ability to present viral glycoproteins at high valency, makes the use of replicating vectors attractive vaccine platforms, particularly for difficult targets such as the HA stem or the HIV Env.

In addition to the duration of replication, another contributor to immunogenicity was inflammation. We observed a modest correlation between the number of reported AEs and the magnitude of the H5-specific neutralizing antibody response. The severity of these was modest, which is consistent with a mild respiratory infection in those who experienced symptoms, and is in the same range of incidence and severity as many replicating and nonreplicating vaccines that are licensed or in development $(19,20)$. The association between inflammation and the magnitude of the immune response also probably contributes to the extraordinary durability of the neutralizing antibody and the efficacy observed following most live virus vaccines (21). High levels of efficacy can also be induced by protein vaccines when combined with newer, highly potent adjuvants. One such example is the Shingrix recombinant glycoprotein $\mathrm{E}$ vaccine adjuvanted with ASO1b that causes systemic symptoms in most vaccinees but is also greater than $90 \%$ effective at preventing shingles $(22,23)$. More modest levels of inflammation may be associated with efficacy in cases where the goal is to prevent viremic spread, such as with the inactivated polio vaccine or the hepatitis A vaccine, or where the targeted virus is directly accessed by high levels of serum $\operatorname{Ig} G$ at the nidus of infection, such as with the human papillomavirus.

In efforts to protect against respiratory pathogens, there are potential concerns regarding the induction of pulmonary inflammation, primarily found in 2 contexts. The first is the direct induction of pulmonary inflammation by the vaccine virus. The immune response is downmodulated by some E3 proteins, such as $\mathrm{p} 19 \mathrm{~K}$, which interferes with MHC class I-restricted antigen presentation, or by the receptor internalization and degradation complex, which interferes with FAS- or TRAIL-mediated apoptosis of infected cells (24). The Ad4-H5-Vtn used in the current study retains these genes, but others with unknown functions are deleted (25). Although E3-deleted mutants of Ad5 may cause some increases in pulmonary pathology in cotton rats, this was not observed in recombinants when the same viruses contained an insert in the Ad4 E3 region $(26,27)$. We did not observe clinical evidence of severe pulmonary inflammation or pneumonia in the current trial. The second concern is the VAED observed upon subsequent infection with the target virus $(11,12)$. In the present study, we observed a balanced $\mathrm{CD}^{+}{ }^{+}$and $\mathrm{CD} 8^{+} \mathrm{T}$ cell response to $\mathrm{H} 5 \mathrm{HA}$ and a signature in serum proteomics consistent with a Th1 response. The drop in Th2 cytokines was further evidence of the skewing toward the more desirable Th1 response, given that these cytokine subsets are counter-regulated. These observations are made with the caveat that the Th2 skew associated with enhanced pathology in experimental animals may be a product of the BALB/c strain of mice in which it was originally described and might occur in the absence of Th2 skewing (11). However, enhanced pathology or enhanced disease has not typically been associated with live virus vaccinations in experimental animals or humans, respectively (12, $28,29)$. It should also be noted that, following a protein boost, we observed a neutralizing antibody response associated with protection that, in addition to the $\mathrm{CD}^{+} \mathrm{T}$ cell response, would further mitigate VAED concerns.
The induction of a mucosal immune response that we observed is potentially a particularly attractive attribute of the intranasal Ad4 platform for respiratory viral pathogens. We observed the induction of H5-specific $\operatorname{IgG}$ and $\operatorname{IgA}$ at each of the mucosal surfaces that were sampled. Although we did not pursue lung biopsies in this study, this immune response was also probably accompanied by the induction of resident memory H5-specific $\mathrm{T}$ cells and $\mathrm{B}$ cells. These responses may play an important role in the protective efficacy of this platform. There are numerous examples of failure to protect against mucosal viral infections using parenterally administered nonreplicating vaccines, among which RSV, parainfluenza virus type 3 (PIV-3), Ad4, rotavirus, and measles vaccines are examples. The reasons for these failures lie, in part, in the difficulty in protecting mucosal surfaces expressing viral receptors on their apical surface; skewed, short-lived $\mathrm{T}$ and $\mathrm{B}$ cell immune responses generated by inactivated vaccines; and the 50- to 1000-fold lower levels of antibodies on these surfaces compared with serum. The use of a cervical cup in this study permitted a direct examination of the level of $\mathrm{H} 5$-specific antibodies in the cervical fluid without the dilution or absorbance onto a swab that limits quantitative analysis by other approaches. In this analysis, we observed a very wide distribution of ratios, but with a median 50-fold lower level of antibodies in the cervical fluid compared with levels in serum. In experimental animals, it takes up to 100 -fold more passively transferred antibodies to protect the URT compared with the lower respiratory tract (30). The lower level of virus-specific IgG at mucosal sites is also potentially consistent with the lower levels in cervical secretions reported in a prior study of HPV vaccination (31). One striking example of the potential for mucosal immunity to contribute to sterilizing immunity is the live-attenuated oral polio vaccine. Upon live vaccine virus challenge, there is sterilizing immunity induced by prior oral polio vaccination, whereas this is not the case for the parenterally administered inactivated vaccine, despite the induction of high levels of serum antibodies (32). It is believed that this induction of sterilizing immunity was a critical attribute of the oral polio vaccine that made it particularly effective in limiting wild-type poliovirus shedding and interrupting its transmission. A similar impact on sterilizing immunity in the URT was recently observed in preclinical work on a SARS-CoV-2 vaccine. In ferrets, intramuscular or mucosal immunization with a replication-defective Ad5-spike recombinant induced similar levels of spike-specific antibodies in the serum, yet only mucosal immunization induced sterilizing protection of the URT (33). A similar advantage of intranasal administration over intramuscular administration in inducing mucosal immunity and sterilizing protection of the URT has recently been observed using lentiviral- or chimp adenoviralspike recombinants in mouse models permissive to SARS-CoV-2 infection (34-36). Of note, local specific IgA has been observed to be highly associated with terminating viral shedding after challenge with coronavirus 229E (37).

There are several important caveats to the immunogenicity of replicating Ad4-H5-Vtn. The first of these is that the degree to which the neutralizing antibody responses observed here will afford protection is unclear. Although the median H5-specific neutralizing antibody titer was 1:1000 even several years after vaccination, it was lower in the MN assay. For H1N1, H2N2, and B viruses, 
an HAI titer above 1:40 has been associated with protection in $50 \%$ of vaccinees for some influenza virus strains (38). Although HAI has been observed to be somewhat insensitive for detecting antibodies against $\mathrm{H} 5$ in humans (39), nearly all participants had HAI titers above the 1:40 level when Ad4-H5-Vtn-primed participants were boosted with protein. Nonetheless, depending on the assay used, the serum neutralizing antibody responses to the licensed vaccine were higher by 1 to 2 logs when preceded by Ad4-H5-Vtn priming. The second caveat to the usefulness of Ad4-H5-Vtn as tested is the diminished response to $\mathrm{H} 5 \mathrm{HA}$ in some participants with preexisting antibodies against Ad4. In this study, we enrolled Ad4-seronegative participants primarily to understand the maximum immunogenicity of the platform. Seropositive participants were enrolled in a separate exploratory arm. This stratification may not be necessary in future studies, and the primary outcome can be focused on the response to the transgene-encoded protein. We were able to infect all participants with the exception of those with the very highest levels of neutralizing antibodies. This is consistent with some prior observations, in which study participants were able to be experimentally reinfected intranasally only weeks to months after a primary infection with Ad4, Ad7, RSV, or coronavirus 229E $(40,41)$. Indeed, one of the strengths of using the URT as a route of administration is that preexisting immunity is relatively inefficient at protecting the URT (30). Although, most participants who were Ad4 seropositive at baseline developed a response to the $\mathrm{H} 5$ transgene, the highest responses were only found in those who were seronegative. This suggests that further circumventing preexisting immunity by the use of other adenovirus serotypes or chimeric Ad4 viruses might be advantageous to the use of this platform. The seroprevalence of antibodies against Ad4 increases with age and may be very low to absent in children 15 years of age or younger (42). The overall immunogenicity of Ad4 recombinants in children may therefore be higher than that reported here. These results also confirmed the results of a prior study demonstrating that this Ad4-H5Vtn vaccine platform is only modestly immunogenic when administered orally (8). This modest immunogenicity is consistent with our more recent study involving 30 participants who received Ad4HIV-Env or -Gag. The wild-type Ad4 and Ad7 oral vaccines given in the military induce a durable neutralizing antibody response and have a protective efficacy against acute respiratory disease of 99\% (43). However, the military example differs from that in the present study in terms of the lack of E3 deletions, the lack of transgene insertions, and the expression of multiple viral determinants that stimulate humoral and cellular immunity against the targeted Ad4 and Ad7 viruses. Although we observed low responses to H5 in recipients of the oral vaccine, this should not be interpreted as a complete lack of priming by this vaccine. In one recent study, a large increase in $\mathrm{H} 5$ antibody responses was observed after administration of an MIV boost following Ad4-H5-Vtn vaccination (14). This was true even among those who did not seroconvert to H5 after receiving oral Ad4-H5-Vtn. It remains possible that in participants whose preexisting immunity may have limited the initial response to URT-administered Ad4-H5-Vtn, the primed response could be similarly boosted. Although orally administered Ad4 recombinants are unlikely to be sufficiently immunogenic as a standalone vaccine, they will likely still have utility when combined with other immunogens in a prime-boost regimen.
One additional consideration regarding the use of replication-competent Ad4-H5-Vtn is the possibility of transmission of the vaccine virus. This may be of particular concern if the virus were transmitted to persons who are immune compromised with regard to virus-specific immunity. Although transmission to household and intimate contacts was not observed here, the numbers of participants are small. There are several factors that may have contributed to the lack of transmission observed. First, respiratory infection with the wild-type Ad4 is thought to require close contact such as that experienced in military barracks. Second, the possibility of transmission was further mitigated by the counseling to avoid intimate contact for 28 days after vaccination. Last, the virus used in the current study was attenuated, likely decreasing the amount and duration of virus shedding compared with the wild-type virus and therefore decreasing the possibility of transmission. Although viral DNA was detected for a much longer period, infectious virus was shed at a low level in only one-third of participants for a median of 1 day. In the end, should this platform prove to be more effective in preventing respiratory viral infections than parenteral nonreplicating vectors, the possibility of transmission of the vaccine virus may need to be balanced against the risk of acquisition and transmission of the more pathogenic target virus, where the level and window of shedding are less well known. Although we have not observed transmission in subsequent trials of Ad4-HIV recombinants, the frequency of transmission to household or intimate contacts is a feature of this vaccine platform that should be monitored in the future.

Taken together, the results of the present study demonstrate the utility of the replicating recombinant Ad4 platform in stimulating a humoral immune response to the transgene as well as the importance of replication in the magnitude and durability of that response. One particular advantage of the platform is the ability of a replicating vector to deliver a large amount of antigen with a very small inoculum. Ad4 recombinants commonly replicate to $10^{11}$ to $10^{13} \mathrm{vp} / \mathrm{mL}$. Given that the infectious dose we observed was $10^{4}$ vp or 1 to 10 infectious units, millions to tens of millions of doses could be produced from relatively small lots. This is a particular advantage for a rapid response to outbreaks such as the current SARS-CoV-2 pandemic. Rather than in vitro production in factories of vast amounts of antigen necessary to immunize large populations, replicating vectors permit this to be accomplished in the vaccinee, in the appropriate conformation and glycosylation state, without the need for characterization and coproduction of adjuvants. Given the results provided here, advantages such as these provide a strong rationale for the use of the Ad4 platform for $\mathrm{H} 5$ influenza viruses - and potentially for other viruses.

\section{Methods}

Study design. Healthy volunteers were enrolled at the NIH under 1 of 2 phase I, single-center, dose-escalation studies. The first clinical protocol (https://clinicaltrials.gov/ct2/show/NCT01443936) recruited subjects for immunization via the oral or tonsillar routes. Intranasal administration was investigated in the second protocol (https://clinicaltrials.gov/ct2/show/NCT01806909).

Individuals meeting the inclusion criteria were screened up to 8 weeks prior to vaccination. Racial and ethnic classifications were recorded at screening from participant self-reporting. The vaccine recipients were selected on the basis of the following criteria: healthy 
men and nonpregnant women, between the ages of 18 and 40 years, with an Ad4 antibody titer $\mathrm{ID}_{80}$ below 100 (Supplemental Figure 1). Ad4-H5-Vtn recombinant vaccine produced by Emergent Biosolutions Inc. is a replication-competent, Ad4-based vaccine carrying a fulllength HA gene from influenza A H5N1 virus (A/Vietnam/1194/2004). Participants in the oral arm of the study received $10^{10} \mathrm{vp}$ of Ad4-H5-Vtn in an enteric-coated capsule $(n=10)$. The other participants were vaccinated during the dose-escalation phase, receiving from $10^{3}$ to $10^{8} \mathrm{vp}$ of Ad4-H5-Vtn, either applied on the tonsils with a cotton swab $(n=25)$ or sprayed intranasally using an Accuspray device (BD Biosciences) ( $n$ $=28$ ). One subject in the tonsillar arm and 2 subjects in the intranasal arm who did not seroconvert after low-dose $\left(10^{3}-10^{4} \mathrm{vp}\right)$ vaccination were revaccinated at a higher dose $\left(10^{7}-10^{8} \mathrm{vp}\right)$. An additional 9 participants who were Ad4 seropositive at screening were dosed by the tonsillar $(n=4)$ or intranasal $(n=5)$ routes in parallel exploratory arms. Only 1 participant in the $10^{8} \mathrm{vp}$ dose group in the tonsillar arm was lost to follow-up after day 21 and was excluded from analysis. There were no major protocol deviations. In an expansion H5N1 boost phase, all vaccinees from the initial phase were offered re-enrollment to receive a booster vaccination with a licensed $\mathrm{H} 5 \mathrm{~N} 1$ inactivated monovalent influenza vaccine. The inactivated monovalent influenza virus vaccine H5N1(A/Vietnam/1203/2004) and the monovalent pandemic recombinant HA influenza vaccine (PanBlok) were produced and provided by Sanofi Pasteur Inc. An additional 5 individuals who had never received an $\mathrm{H} 5$ influenza vaccine were enrolled as controls.

Detection of viral shedding. Viral DNA was extracted from nasal, rectal, or throat swabs using the KingFisher Flex Purification System (Thermo Fisher Scientific). Standards were created by diluting aliquots of Ad4-H5-Vtn vaccine quantified by HPLC. qPCR was performed using the QuantStudio 3 System (Thermo Fisher Scientific) to measure the levels of viral DNA in nasal, rectal, or throat swabs as previously described (25). DNA extract $(6.25 \mu \mathrm{L})$ was combined with TaqMan Fast Advanced Master Mix (Thermo Fisher Scientific). The reaction conditions were as follows: one 20 second period at $95^{\circ} \mathrm{C}$, followed by 40 cycles of 1 second at $95^{\circ} \mathrm{C}$ and 20 seconds at $60^{\circ} \mathrm{C}$. The $\mathrm{H} 5$-Vtn insert was amplified using the following primer and probe sets: forward primer 5'-CAGCTTGTGCACGGTGCTAA-3', reverse primer 5'-TGCAAAAAGAAGCACTATTTTCTC C-3', and probe 5'-/56-FAM/CGCGATCGTGTGCCTGAGCATTC/36-TAMSp/-3'. Replication-competent virus in nasal swab elutants was measured by an ELISA with the AdEasy Viral Titer Kit (Agilent Technologies).

$T$ cell responses. Intracellular cytokine assays were used to enumerate $\mathrm{H} 5$-specific $\mathrm{T}$ cells in PBMC samples from vaccine recipients obtained before vaccination and at week 4 after vaccination, as described previously (44). Briefly, thawed PBMCs were cultured overnight prior to stimulation with medium alone, phorbol myristate acetate (6.5 nM; Calbiochem) plus ionomycin (0.2 $\mu \mathrm{M}$; MilliporeSigma), or a 93-peptide array of mostly 17 mers with 11 amino acid overlaps spanning the HA protein of the A/Vietnam/1203/2004 (H5N1) strain of influenza virus (BEI Resources, ref. 44)

PVEI, MN, and HAI assays. HA-pseudotyped lentiviruses were generated as previously described (45). Ad4-neutralizing antibodies were measured using a luciferase-based Ad4 virus as previously described (9). In the PVEI assay, H5-specific neutralizing antibodies were measured using a pseudotyped lentivirus luciferase-based assay as previously described (9). MN and HAI assays were performed as previously described (9).
SOMAscan assay and proteomics data analysis. Proteomics profiles were characterized using the SOMAscan Assay 1.3k (SomaLogic). The basis of the SOMAscan is built on the use of a new generation of protein-capture slow off-rate modified aptamer (SOMAmer) reagents (46). The assay consists of 1305 SOMAmer reagents selected against a variety of human proteins that belong to broad biological subgroups. Runs in the 1.3k Assay were performed semiautomatically with a Tecan Freedom Evo 200 High-Throughput System. Quality control and calibrators are pooled samples composed of the same matrix as the biological samples being measured in the plate. Following standard data normalization procedures (47), raw data were first transformed by hybridization control normalization, which utilizes 12 spike-in SOMAmer controls to remove well-to-well variance in the hybridization process, and then data were normalized across plates using calibrator samples, followed by median signal normalization to remove intraplate variance due to sample-to-sample differences in loading volume, leaks, washing conditions, etc. An interactive Shiny web tool (48) was used during the quality control process at every step of the data normalization process.

Detection of H5-specific antibodies in serum and mucosal secretions. Nasal and rectal sponge wicks were transferred to SPIN-X columns with no filter (MilliporeSigma) and then soaked with $300 \mu \mathrm{L}$ elution buffer (PBS, 0.25\% BSA, with cOmplete Mini EDTA-free Protease Inhibitor [Roche Diagnostics]) for 10 minutes. Tubes were then spun at $19,000 g$ for 5 minutes. Flow-through was transferred to a new SPIN-X column with a $0.22 \mu \mathrm{m}$ filter (MilliporeSigma) and spun at 21,130 $\mathrm{g}$ for 20 minutes. Cervical cups were thawed on ice and then spun at $400 \mathrm{~g}$ at $4^{\circ} \mathrm{C}$ for 20 minutes. Flow-through was diluted 1:1 by volume with elution buffer. A/Vietnam/1203/2004 H5 HA protein (eEnzyme) was coupled prior to the experiment to Bio-Plex Pro Magnetic $\mathrm{COOH}$ Beads (Bio-Rad) according to the manufacturer instructions. Samples were run on the Bio-Plex 200 System according to the manufacturer's instructions. Briefly, for the measurement of H5-specific IgG and IgA, A/Vietnam/1203/2004 H5 HA protein, and 1626 H5 HA-coupled beads were incubated with a $50 \mu \mathrm{L}$ sample diluted in sample diluent on a shaker (900 rpm) for 1 hour. The beads were washed 3 times using the automated plate washer EL $\times 405$ Select CW (BioTek) and then incubated with $25 \mu \mathrm{L}$ biotinylated secondary anti-human IgG or IgA antibody in diluent buffer on a shaker (900 rpm) for 30 minutes. The beads were washed again and then incubated with $50 \mu \mathrm{L}$ phycoerythrin-conjugated (PE-conjugated) streptavidin on a shaker (900 $\mathrm{rpm}$ ) for 1 hour. The beads were washed again and then incubated with $125 \mu \mathrm{L}$ assay buffer on a shaker for 30 seconds. Anti-H5 HA monoclonal IgG or IgA antibodies with a known concentration were used as standards. For the measurement of total IgG and IgA, the Bio-Plex Pro Human Isotyping Assay Kit (Bio-Rad) was used to run a 6-plex assay. The proportions of $\mathrm{H} 5$-specific $\operatorname{IgG}_{1}$ and IgA were calculated from the results of the H5-specific single-plex and total Ig 6-plex assays.

Statistics. For comparisons of 2 groups, we used the 2-sample $t$ test assuming unequal variances. When comparing 2 groups with each other, where each sample from 1 group could be paired with another sample from the second group, we used the paired $t$ test. For comparisons of 2 groups, where individuals had longitudinal measurements (e.g., comparison of Ad4 neutralization titers between oral versus tonsillar groups, Figure 2B), to account for correlations over time for each individual, we used generalized estimating equations with an autoregressive correlation structure; week 0 measurements were not 
included. When comparing AE percentages, the Fisher's exact test was performed. All tests were 2 sided. All analyses were conducted in $\mathrm{R}$ or GraphPad Prism (GraphPad Software). For multiple-comparison analyses, the Benjamini-Hochberg procedure was used to estimate the FDR rate at a fixed $P$ value significance threshold of 0.05 for each set of tests (49). For the SOMAscan data, relative abundances for selected target proteins were compared for different subject groups and time points using Wilcoxon-Mann-Whitney $U$ (signed-rank) tests after normalization. The analysis was implemented using custom scripts in $\mathrm{R}$ (version 4.0.2).

Study approval. Ad4-H5-Vtn vaccine recipients were enrolled at the NIH under a clinical protocol approved by the IRB of the NIAID (ClinicalTrials.gov NCT01443936). All vaccine recipients and their household contacts provided written informed consent in accordance with the Declaration of Helsinki.

\section{Author contributions}

$\mathrm{KM}, \mathrm{SAM}, \mathrm{JH}, \mathrm{JS}, \mathrm{JA}, \mathrm{MG}$, and MC conceived, designed, and coordinated the study. SAM and A. Poole executed the clinical study. BHK, EI, TG, SS, AAP, LB, JH, SE, JP, NK, DS, NW, MZ, TS, A. Patamawenu, and KMM performed immunogenicity studies. JC, AB, and GF performed the SOMAscan sera measurements and analyses. DS and FK conceived and performed MN and HAI assays. CJL performed the statistical analyses. KM, SAM, JH, TG, SS, LB, NW, $\mathrm{FK}$, and MC contributed to the writing and revision of the manuscript. All authors read and approved the manuscript.

\section{Acknowledgments}

We would like to thank Ruvim Izikson, Tim Alefantis, Manon Cox, Jonathan Haines, and Jim Tartaglia from Sanofi Pasteur for providing the PanBlok and Sanofi MIV vaccines used in this study. The peptide array, influenza virus A/New York/348/2003 (H1N1) matrix protein 1, NR-2613 reagent was obtained through the BEI Resources Repository of the NIAID, NIH. Support for this work was provided by the Intramural Research Program of the NIAID, NIH. Funding for the Krammer laboratory was provided by a grant from the NIAID, NIH (U19 AI109946) and the CEIRS, NIAID, NIH (contract HHSN272201400008C).

Address correspondence to: Mark Connors, NIAID, LIR, HSIS, Bldg. 10, Rm 11B07c, 10 Center Drive, Bethesda, Maryland 20892, USA. Phone: 301.496.8057; Email: mconnors@nih.gov.
1. Gerdil C. The annual production cycle for influenza vaccine. Vaccine. 2003;21(16):1776-1779.

2. Robert-Guroff M. Replicating and non-replicating viral vectors for vaccine development. Curr Opin Biotechnol. 2007;18(6):546-556.

3. Cirelli KM, et al. Slow delivery immunization enhances HIV neutralizing antibody and germinal center responses via modulation of immunodominance. Cell. 2019;177(5):1153-1171.

4. Gaydos CA, Gaydos JC. Adenovirus vaccines in the US military. Mil Med. 1995;160(6):300-304.

5. Smith TJ, et al. Experimental respiratory infection with type 4 adenovirus vaccine in volunteers: clinical and immunological responses. J Infect Dis. 1970;122(4):239-248.

6. Couch RB, et al. Aerosol-induced adenoviral illness resembling the naturally occurring illness in military recruits. Am Rev Respir Dis. 1966;93(4):529-535

7. Patterson LJ, et al. Protection against mucosal simian immunodeficiency virus SIV (mac251) challenge by using replicating adenovirus-SIV multigene vaccine priming and subunit boosting. J Virol. 2004;78(5):2212-2221.

8. Gurwith M, et al. Safety and immunogenicity of an oral, replicating adenovirus serotype 4 vector vaccine for $\mathrm{H} 5 \mathrm{~N} 1$ influenza: a randomised, double-blind, placebo-controlled, phase 1 study. Lancet Infect Dis. 2013;13(3):238-250.

9. Matsuda K, et al. Prolonged evolution of the memory B cell response induced by a replicating adenovirus-influenza $\mathrm{H} 5$ vaccine. Sci Immunol. 2019;4(34):eaau2710.

10. Ludwig SL, et al. Prevalence of antibodies to adenovirus serotypes 4 and 7 among unimmunized US Army trainees: results of a retrospective nationwide seroprevalence survey. J Infect Dis. 1998;178(6):1776-1778.

11. Haynes BF, et al. Prospects for a safe COVID-19 vaccine. Sci Transl Med. 2020;12(568):eabe0948.

12. Acosta PL, et al. Brief history and characteriza- tion of enhanced respiratory syncytial virus disease. Clin Vaccine Immunol. 2015;23(3):189-195.

13. Renegar KB, et al. Role of IgA versus IgG in the control of influenza viral infection in the murine respiratory tract. JImmunol. 2004;173(3):1978-1986.

14. Khurana S, et al. Oral priming with replicating adenovirus serotype 4 followed by subunit H5N1 vaccine boost promotes antibody affinity maturation and expands $\mathrm{H} 5 \mathrm{~N} 1$ cross-clade neutralization. PLoS One. 2015;10(1):e0115476.

15. Malherbe DC, et al. Combination adenovirus and protein vaccines prevent infection or reduce viral burden after heterologous clade $\mathrm{C}$ simian-human immunodeficiency virus mucosal challenge. J Virol. 2018;92(2):e01092-17.

16. Talaat $\mathrm{KR}$, et al. A live attenuated influenza $\mathrm{A}(\mathrm{H} 5 \mathrm{~N} 1)$ vaccine induces long-term immunity in the absence of a primary antibody response. J Infect Dis. 2014;209(12):1860-1869.

17. Carnell GW, et al. Pseudotype-based neutralization assays for influenza: a systematic analysis. Front Immunol. 2015;6:161.

18. Chen WH, et al. Persistence of antibody to influenza A/H5N1 vaccine virus: impact of ASO3 adjuvant. Clin Vaccine Immunol. 2016;23(1):73-77.

19. Bahl K, et al. Preclinical and clinical demonstration of immunogenicity by mRNA vaccines against $\mathrm{H} 10 \mathrm{~N} 8$ and H7N9 influenza viruses. $\mathrm{Mol}$ Ther. 2017;25(6):1316-1327.

20. Moa AM, et al. Immunogenicity and safety of inactivated quadrivalent influenza vaccine in adults: A systematic review and meta-analysis of randomised controlled trials. Vaccine. 2016;34(35):4092-4102.

21. Amanna IJ, et al. Duration of humoral immunity to common viral and vaccine antigens. $\mathrm{NEnglJ}$ Med. 2007;357(19):1903-1915.

22. Heineman TC, et al. Understanding the immunology of Shingrix, a recombinant glycoprotein $\mathrm{E}$ adjuvanted herpes zoster vaccine. Curr Opin Immunol. 2019;59:42-48.
23. Cohen JI. A new vaccine to prevent herpes zoster. N Engl J Med. 2015;372(22):2149-2150.

24. Lichtenstein DL, et al. Functions and mechanisms of action of the adenovirus E3 proteins. Int Rev Immunol. 2004;23(1-2):75-111.

25. Alexander J, et al. Pre-clinical evaluation of a replication-competent recombinant adenovirus serotype 4 vaccine expressing influenza $\mathrm{H} 5$ hemagglutinin. PLoS One. 2012;7(2):e31177.

26. Ginsberg HS, et al. Role of early region 3 (E3) in pathogenesis of adenovirus disease. Proc Natl Acad Sci U S A. 1989;86(10):3823-3827.

27. Patterson LJ, et al. Insertion of HIV-1 genes into Ad4DeltaE3 vector abrogates increased pathogenesis in cotton rats due to E3 deletion. Virology. 2002;292(1):107-113.

28. Polack FP. Atypical measles and enhanced respiratory syncytial virus disease (ERD) made simple. Pediatr Res. 2007;62(1):111-115.

29. Connors M, et al. Cotton rats previously immunized with a chimeric RSV FG glycoprotein develop enhanced pulmonary pathology when infected with RSV, a phenomenon not encountered following immunization with vaccinia-RSV recombinants or RSV. Vaccine. 1992;10(7):475-484.

30. Siber GR, et al. Comparison of antibody concentrations and protective activity of respiratory syncytial virus immune globulin and conventional immune globulin. J Infect Dis. 1994;169(6):1368-1373.

31. Scherpenisse M, et al. Detection of systemic and mucosal HPV-specific IgG and IgA antibodies in adolescent girls one and two years after HPV vaccination. Hum Vaccin Immunother. 2013;9(2):314-321.

32. Hird TR, Grassly NC. Systematic review of mucosal immunity induced by oral and inactivated poliovirus vaccines against virus shedding following oral poliovirus challenge. PLoS Pathog 2012;8(4):e1002599.

33. Wu S, et al. A single dose of an adenovirus-vectored vaccine provides protection against SARS- 
CoV-2 challenge. Nat Commun. 2020;11(1):4081.

34. $\mathrm{Ku} \mathrm{M}-\mathrm{W}$, et al. Intranasal vaccination with a lentiviral vector strongly protects against SARS-CoV- 2 in mouse and golden hamster preclinical models [preprint]. https://doi. org/10.1101/2020.07.21.214049. Posted on bioRxiv September 3, 2020.

35. Hassan AO, et al. A single-dose intranasal ChAd Vaccine protects upper and lower respiratory tracts against SARS-CoV-2. Cell. 2020;183(1):169-184.

36. King RG, et al. Single-dose intranasal administration of AdCOVID elicits systemic and mucosal immunity against SARS-CoV-2 in mice [preprint]. https://doi. org $/ 10.1101 / 2020.10 .10 .331348$. Posted on bioRxiv October 11, 2020.

37. Callow KA. Effect of specific humoral immunity and some non-specific factors on resistance of volunteers to respiratory coronavirus infection. JHyg (Lond). 1985;95(1):173-189.
38. Krammer F, et al. Meeting report and review: immunological assays and correlates of protection for next-generation influenza vaccines. Influenza Other Respir Viruses. 2020;14(2):237-243.

39. Rowe $\mathrm{T}$, et al. Detection of antibody to avian influenza A (H5N1) virus in human serum by using a combination of serologic assays. JClin Microbiol. 1999;37(4):937-943.

40. Callow KA, et al. The time course of the immune response to experimental coronavirus infection of man. Epidemiol Infect. 1990;105(2):435-446.

41. DeVincenzo JP, et al. Viral load drives disease in humans experimentally infected with respiratory syncytial virus. Am J Respir Crit Care Med. 2010;182(10):1305-1314.

42. Jordan WS Jr. Occurrence of adenovirus infections in civilian populations. AMA Arch Intern Med.1958;101(1):54-59.

43. Collins ND, et al. Live oral adenovirus type 4 and type 7 vaccine induces durable antibody response. Vaccines (Basel). 2020;8(3):E411.
44. Migueles SA, et al. Defective human immunodeficiency virus-specific CD8+ T-cell polyfunctionality, proliferation, and cytotoxicity are not restored by antiretroviral therapy. J Virol. 2009;83(22):11876-11889.

45. Naldini L, et al. In vivo gene delivery and stable transduction of nondividing cells by a lentiviral vector. Science. 1996;272(5259):263-267.

46. Rohloff JC, et al. Nucleic acid ligands with protein-like side chains: modified aptamers and their use as diagnostic and therapeutic agents. Mol Ther Nucleic Acids. 2014;3:e201.

47. Candia J, et al. Assessment of variability in the SOMAscan assay. Sci Rep. 2017;7(1):14248.

48. Cheung F, et al. Web tool for navigating and plotting somalogic ADAT files. JOpen Res Softw. 2017;5:20.

49. Benjamini Y, Hochberg Y. Controlling the false discovery rate - a practical and powerful approach to multiple testing. JR Stat Soc B. 1995;57(1):289-300. 